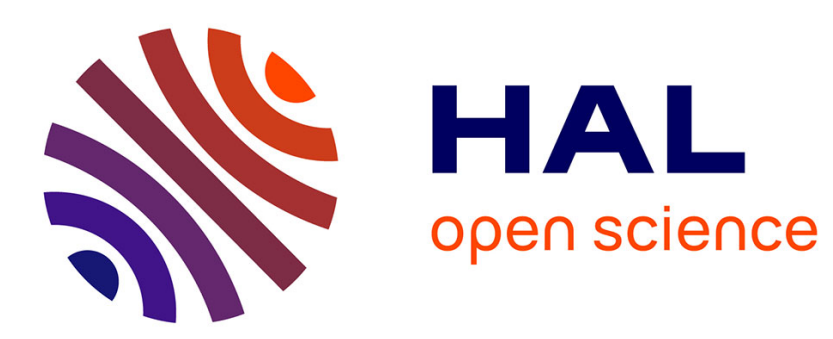

\title{
Granularity in relational formalisms with application to time and space representation
}

Jérôme Euzenat

\section{To cite this version:}

Jérôme Euzenat. Granularity in relational formalisms with application to time and space representation. Computational Intelligence, 2001, 17 (4), pp.703-737. 10.1111/0824-7935.00170 . hal-00822915

\section{HAL Id: hal-00822915 \\ https://hal.inria.fr/hal-00822915}

Submitted on 15 May 2013

HAL is a multi-disciplinary open access archive for the deposit and dissemination of scientific research documents, whether they are published or not. The documents may come from teaching and research institutions in France or abroad, or from public or private research centers.
L'archive ouverte pluridisciplinaire HAL, est destinée au dépôt et à la diffusion de documents scientifiques de niveau recherche, publiés ou non, émanant des établissements d'enseignement et de recherche français ou étrangers, des laboratoires publics ou privés. 


\title{
GRANULARITY IN RELATIONAL FORMALISMS - WITH APPLICATION TO TIME AND SPACE REPRESENTATION
}

\author{
JÉRÔME EUZENAT \\ INRIA Rhône-Alpes, Montbonnot, France
}

\begin{abstract}
Temporal and spatial phenomena can be seen at a more or less precise granularity, depending on the kind of perceivable details. As a consequence, the relationship between two objects may differ depending on the granularity considered. When merging representations of different granularity, this may raise problems. This paper presents general rules of granularity conversion in relation algebras. Granularity is considered independently of the specific relation algebra, by investigating operators for converting a representation from one granularity to another and presenting six constraints that they must satisfy. The constraints are shown to be independent and consistent and general results about the existence of such operators are provided. The constraints are used to generate the unique pairs of operators for converting qualitative temporal relationships (upward and downward) from one granularity to another. Then two fundamental constructors (product and weakening) are presented: they permit the generation of new qualitative systems (e.g. space algebra) from existing ones. They are shown to preserve most of the properties of granularity conversion operators.

Key words: Granularity, space representation, time representation, relation algebra, interval algebra, product, weakening.
\end{abstract}

\section{INTRODUCTION}

"Imagine that you are biking in a flat countryside. At some distance ahead of you there is a truck parked. You are just able to say (a) that a truck (T) is parked beside a house $(\mathrm{H})$, it seems that they touch each other. When you come closer (b) you are able to distinguish a bumper (B) between them, and even closer (c), you can perceive the space between the bumper and the house."

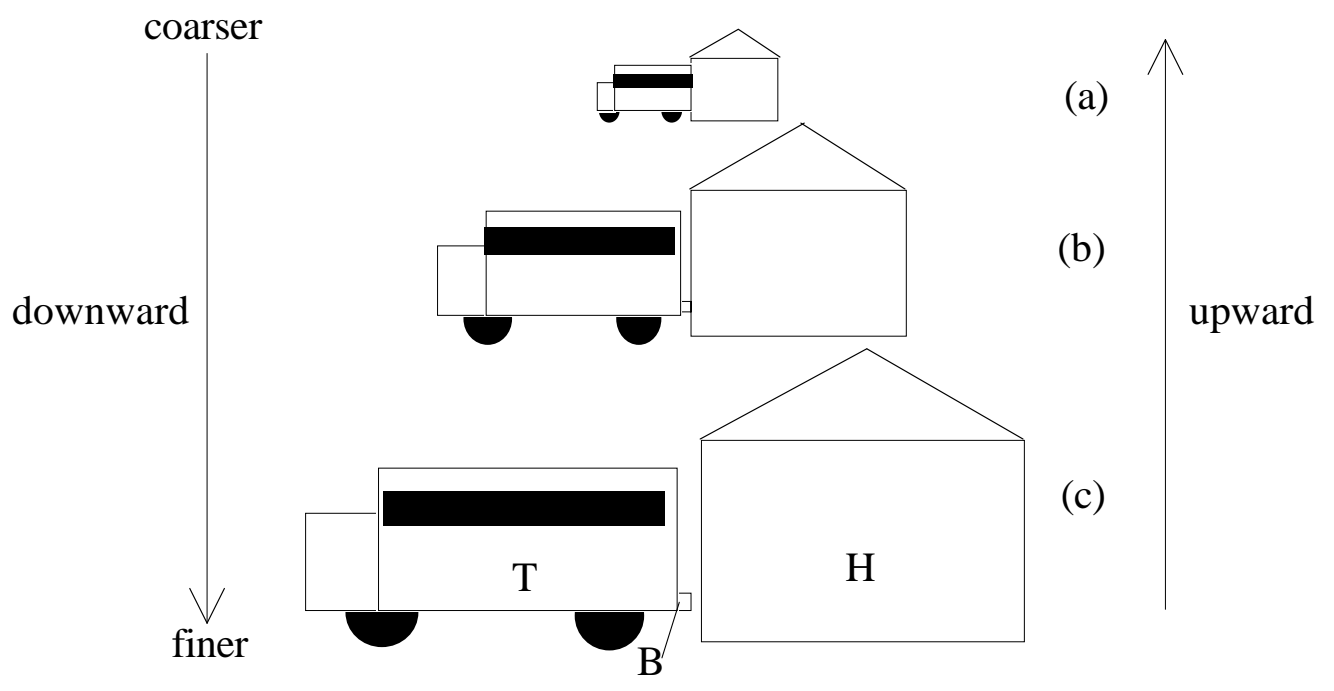

Figure 1. The same scene at three different granularities. It is taken as a spatial metaphor for granularity and is used throughout the paper.

This example shows the description of the same reality perceived at several levels or granularities. It can be similarly applied to time: imagine an agent gathering calendar information on a network with a granularity of days. The agenda states that a particular person is in Grenoble from Monday to Tuesday and in Boston from Wednesday to Friday. If another agent, attached to a second person has the 
information that the two persons will meet on Tuesday, $6 \mathrm{pm}$ in Paris, how can the apparently contradictory information be merged? Now the assistant of the first person has got more precise data: (s)he will be in Grenoble until Tuesday at 10am, in the TGV train from $10 \mathrm{am}$ to $3 \mathrm{pm}$, in Paris from $3 \mathrm{pm}$ to $7 \mathrm{pm}$ and in the plane from Paris to Boston from 11pm to 3am (local time) on Wednesday.

Another application concerns the generation of geographic maps. When considering a particular field map, one can see that it generally contains several maps: the precise one (the one you bought), a smaller map of the same area on the cover which shows the main sites contained in the map and an even smaller one on the back showing how the present map relates to the other maps of the same collection. Obviously these maps are not mere projections, at different scales, of the same huge universal map. They are representations of the same reality, but the representation process went through different filters, which made some details vanish, and through different formalisms, which transformed the polygon representing a particular town on the precise map into a point on the intermediate one and then nothing on the coarsest one.

Different individuals, institutions, etc. use various granularities and, moreover, people communicate data expressed at different granularities. Hence it is fundamental to guarantee the consistency of representations and processes involving different granularities. As a matter of fact, there could be a problem if, in the example of figure 1, someone at position (a) asked "how would you call what is between $\mathrm{H}$ and T?" because at that granularity, the description of the scene would assume that there is nothing between $\mathrm{H}$ and $\mathrm{T}$.

Temporal and spatial granularity is involved in the fusion of knowledge provided by sources of different resolutions (for instance, agents - human or computers communicating about the same situation). It can also be used for structuring a reasoning process by drawing inference at the right level of resolution (in the example of figure 1, from the standpoint of the observer, the granularity (a) is informative enough for deciding that the truck driving wheel is on the left of the house). Granularity is applied in many domains, like planning (Badaloni\& 1994), cadastral and digital representation (Olivier\& 1995; Papadias\& 1995), geographical information systems (Egenhofer\& 1991; Grigni\& 1995), program specification and proof (Ciapessoni\& 1993; Fiadeiro\& 1994), databases (Dyreson\& 1995, Bettini\& 1998b), and scene and story understanding (Euzenat 1993, Becher\& 2000).

The study of granular knowledge representation thus tries to express how the same phenomenon can be consistently expressed in different manners at different granularities. Being able to deal with granularity corresponds to being able to carry out a set of operations:

- converting a representation from one granularity to another one (how could a particular representation appear at a finer or coarser granularity?);

- testing the compatibility of two representations (is it possible that they represent the same situation at different granularities?);

- localizing the relative granularities of two representations (which of the two given representations of a particular situation can be the coarser one?).

Granularity conversion can be achieved through operators, which, from a situation expressed at a particular granularity, predict how it is perceivable at another granularity.

Granularity is considered here in the context of relational formalisms, i.e. when the representation language is restricted to the representation of relationships holding between entities. Such formalisms provide only a qualitative representation of the situation but they are widespread as they cover relation algebras for dealing with 
time (Allen 1983; Vilain\& 1986) and space (Güsgen 1989; Egenhofer\& 1992; Randell\& 1992a). At the best of our knowledge, granularity in relation algebras has never been studied in a systematic way before (Euzenat 1993; Euzenat 1995a). In (Hobbs 1985), granularity is expressed between logical theories and does not depend on a structuring dimension like time or space. The many other contributions that have dealt with time granularity have focussed on quantitative granularity only (see "Related work" below).

The present paper introduces a general framework for defining granularity conversion operators in (extended) relation algebras and provides general results about the existence of such operators. Furthermore, it produces granularity conversion operators for the relation algebras cited above by applying this framework. It also establishes specific results about the uniqueness of operators for particular formalisms and the distributivity of conversion over relation composition (i.e. inference).

However, the results are restricted to homogeneous representations (i.e. when the representation at each granularity is represented in the same language) and does not account for vanishing objects. For instance, the bumper (B) of figure 1, is not visible at (a) so it has no relation with any other objects: we do not try to decide what happened to the bumper but what happens to the relationships between the remaining objects.

The paper is organized as follows: A first section (\$2) provides the basic definitions of the formalisms (extended relation algebra) that will be used. Such formalisms are widely used in artificial intelligence (for instance, the Allen's interval algebra is an extended relation algebra).

Then, the notion of granularity conversion operators in these extended relational algebras is discussed (\$3). First, the expected nature and form of such operators are considered; then, a set of six constraints stated as relevant to granularity conversion is provided. These constraints are very important because, once accepted, they entails the other results. The first general results about existence and non-existence of granularity conversion operators are then given: there are small algebras, without trivial operators, and characterized situations, in which operators satisfying the constraints can be designed.

The two following sections are devoted to the application of the general constraints about granularity conversion to time and space relation algebras. The application to time algebras $(\$ 4)$ begins with the generation of the unique couple of operators satisfying the core constraints for the instant algebra. It then shows how these results are directly transferred to the interval algebra with the help of the sixth constraint. As a result, the unique granularity conversion operators are provided for two widely used formalisms. Additional results in this section concern the relationship between conversion and composition of relations which had not been explored in the general framework.

The application to space representation formalisms (\$5) uses another method: it provides constructors, Cartesian product and weakening, that can transform one algebra into another. These constructors are interesting because they can be used for generating space representation formalisms. The product constructor is shown to preserve all the constraints for conversion operators (including the properties relating conversion to composition). It is thus able to generate new algebras with their granularity conversion operators. The weakening constructor only preserves four out of six constraints, but it is shown that it can nevertheless generate valid granularity conversion operators when weakening the interval algebra.

The results of $\S 4$ have been published in (Euzenat 1995a) in a less general context. The proofs of the propositions 4 to $10(\$ 4)$ are provided by exhaustive 
check in (Euzenat 1994), those of propositions 11 to 13 (\$5) are provided axiomatically in (Euzenat 1994) and those of propositions 1, 2, 3, 11, 12, 13 and 15 are given in appendix B.

\section{RELATIONAL FORMALISMS}

The model of granularity presented here applies to particular formalisms that will be called extended relation algebras. They allow one to represent the situation given in the introduction by means of suitable binary relationships. For instance, the fact that the bumper touches the house in situation (b) can be expressed as the relation "touch" between the entities B and $\mathrm{H}$. These extended relation algebras are introduced in the first part of the section. Each of them consists of a binary relation algebra provided with a neighborhood relation. Such structures are heavily used for representing time and space. In the remainder, examples will be provided in the context of time representation.

\subsection{Extended relation algebras}

An algebra of binary relations (hereafter referred to as relation algebra) (Tarski $1941)$ is a structure $\left\langle\mathrm{A}, \wedge, \vee,{ }^{*}, 1,0,1, \neg\right\rangle$ in which: $\langle\mathrm{A}, \wedge, \vee, 1,0\rangle$ is a Boolean algebra; * is an associative internal composition law with (left and right) unity element 1 ', that distributes over $\vee ; \neg$ is an internal involutive unary operator, that distributes over $\wedge$, $\checkmark$ and $*$. The extension considered here consists in adding a neighborhood structure to such an algebra.

The present work is concerned with a particular type of relation algebras in which A is the powerset of a generating set $\Gamma$ closed under $\neg$ (hereafter ${ }^{-1}$ ) and $N / \vee$ are set intersection/union $(\cap / \cup)$. Such relation algebras are denoted by $\left\langle 2^{\Gamma}, \cap, \cup, \mathrm{o}, \Gamma,\{\}, e^{-1}{ }^{-1}\right.$. If $\Gamma$ is thought of as the set of possible binary relations between the entities of a particular domain, the use of sets of relations allows one to express the lack of knowledge about the exact relationship between two entities by expressing a disjunction of possible relations. $o$ is the composition between these relations and ${ }^{-1}$ provides the converse relation. These operations are applied to sets of relations by distributing them on each element.

As an example, if $\Gamma$ is reduced to three basic relations $\langle$,$\rangle and =$, it is possible to express that $x$ is before of equal to $y$ by $x\{<=\} y$.

The concept of relation algebra is extended by considering a neighborhood structure on the generating set (Nökel 1988, Freksa 1992). A neighborhood $N$ is a binary reflexive and symmetrical relation over the set $\Gamma$. It is usually represented as a non-oriented graph between the relations. Two qualitative relations between two entities are called conceptual neighbors if they can be transformed one into another through a continuous deformation of the situation (Freksa 1992). A conceptual neighborhood is a set of relations whose elements constitute a connected sub-graph of the neighborhood graph (figure 2). 
DEFINITION (conceptual neighbor relationship): The conceptual neighbor relationship is a binary relation $N_{\Gamma}^{X}$ on a set $\Gamma$ of relations such that $N_{\Gamma}^{X}\left(r, r^{\prime}\right)$ if and only if the continuous transformation $X$ of a situation involving an entity $x$ in relationship $r$ with another entity $y$ can put them in relation $r$ ' without any transition through other relations.

The notation $N_{\Gamma}^{X}(r)$ is used for denoting the set of $r$ 's neighbors. The graph of figure 2a represents the graph of conceptual neighborhood $N_{3}^{A}$ between instants (the only one-dimensional continuous deformation is translation of one instant). The graph of figure $2 \mathrm{~b}$ represents the conceptual neighborhood $N_{13}^{A}$ for the deformation corresponding to the shift of one endpoint of one of the two intervals (as far as it remains a valid interval, i.e. the beginning is strictly before the end). More generally, the deformation corresponds to moving a limit. Throughout the paper, the only transformation considered, $\mathrm{A}$, is the continuous move of a limit (called Aneighborhood in (Freksa 1992)). The consequences of this restriction are acknowledged when important.

The general notion (i.e. not bound to a particular representation) of conceptual neighborhood has not been formally defined so far. It has initially been put forth informally by providing a few examples of continuous transformations that make sense. The generalization is however justified because the idea has been found useful in various contexts (e.g. transitivity table compaction (Freksa, 1992) or transitivity table computation (Randell\& 1992b)) for various representations of space and time.

In the following, we shall focus on extended relation algebras. They are made of a relation algebra generated by a set $\Gamma$ provided with a neighborhood relation. Two examples of such extended relation algebras used for representing time are given below.

\subsection{Instant (point) algebra}

An instant is a durationless temporal entity (also called time point, by analogy with a point on a line; the term point algebra is not used here because it can be misleading in the context of space representation). It can be numerically represented by a date. A relational representation involving instants requires identifying the considered instants and their relationships. There are three possible mutually exclusive relationships between instants. They are called "before" (<), "after" $(>)$ and "simultaneously" (=). The set $\{<,=,>\}$ is called $A_{3}$.

\begin{tabular}{|c|c|c|}
\hline relation $(\mathrm{r}): x \mathrm{r} y$ & $x / y$ & converse: $y r^{-1} x$ \\
\hline before $(<)$ & 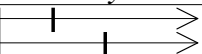 & after $(>)$ \\
\hline simultaneously (=) & $1>$ & $=$ \\
\hline
\end{tabular}

Table 1. The 3 relationships between instants $x$ and $y$.

The composition operation allows one to deduce the relationship between two instants $x$ and $z$, even if it has not been stated, by propagating the known relationships involving intermediate instants. For instance, if $x$ is simultaneous $(\{=\})$ to $y$ which is anterior $(\{<\})$ to $z$, then $x$ is anterior to $z$; this operation is called composition of temporal relations. The composition operator $x_{3}$ is represented by a composition table (table 2 ) which indeed indicates that $=x_{3}<$ yields $\{<\}$. 


\begin{tabular}{c|ccc}
$\times 3$ & $>$ & $=$ & $<$ \\
\hline$>$ & $>$ & $>$ & $<=>$ \\
$=$ & $>$ & $=$ & $<$ \\
$<$ & $<=>$ & $<$ & $<$
\end{tabular}

Table 2. Composition table between instant relationships.

The neighborhood relation in $\mathrm{A}_{3}$ corresponds to the effect of continuously moving instants in time. It is depicted in figure $2 \mathrm{a}$.

For instance, if the example of figure 1 is modeled through bounding instants $\left(x^{-}\right.$ for the left endpoint and $x^{+}$for the right endpoint) of intervals $\mathrm{T}^{+}, \mathrm{B}^{-}, \mathrm{B}^{+}$and $\mathrm{H}^{-}$, it is represented in (c) by $\mathrm{T}^{+}=\mathrm{B}^{-}$(the truck ends where the bumper begins), $\mathrm{B}^{-}<\mathrm{B}^{+}$ (the beginning of the bumper is before its end), $\mathrm{B}^{+}<\mathrm{H}^{-}$(the end of the bumper is before the beginning of the house). One can deduce that the (right extremity of the) truck is left of the (left extremity of the) house because $\mathrm{T}^{+}=\mathrm{B}^{-}, \mathrm{B}^{-}<\mathrm{B}^{+}, \mathrm{B}^{+}<\mathrm{H}^{-}$, and $=\mathrm{X}_{3}<$ gives $\{<\}$ and $<$ composed with $<$ yields $\{<\}$ again. A continuous modification of situation (c) towards situation (b) is possible (by enlarging the bumper). As a matter of fact, the bumper can progressively be enlarged (without changing the qualitative description of the situation) as far as it does not touch the house. Once it meets the house, the situation is changed to situation (b).

\subsection{Interval algebra}

The interval algebra (Allen 1983) is another well known algebra of relations. An (uninterrupted) period is a temporal entity with a duration. It can be thought of as a segment on a straight line. A numerical representation of a period is an interval: a couple of bounds (beginning instant, ending instant) or a beginning instant and a duration. Intervals can be manipulated through a set of 13 mutually exclusive temporal relationships between two intervals (see table 3); this set is called $\mathrm{A}_{13}$.

relation (r): $x \mathrm{r} y$
before (b)
during (d)
overlaps (o)
starts (s) (and finishes before)
finishes (f) (and start after)
meets (m)
equals (e)

Table 3 (from (Allen 1983)). The 13 relationships between two intervals $x$ and $y$.

The composition operator $\times_{13}$ is represented by a composition table (Allen 1983), similar to the table 2 .

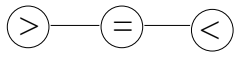

(a)

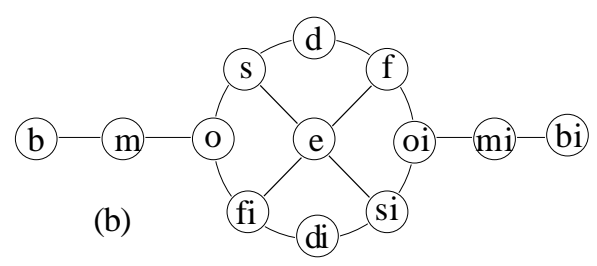


Figure 2. Neighborhood graphs for (a) instant-to-instant relations, (b) interval-to-interval relations (from (Nökel 1988)). The neighborhood graph is made of relations as nodes and conceptual neighborhood as edges (converse relationships are denoted with an "i" added at the end for the sake of readability).

For instance, the three situations of figure 1 can be expressed in the same formalism with objects and qualitative relations between them. Provided that only the positions of the objects along the horizontal line are considered, the three elements $(T, B$ and $H)$ are related to each other in the way of figure $1 \mathrm{c}$ by $T\{m\} B$ (the truck meets its bumper) and $\mathrm{B}\{\mathrm{b}\} \mathrm{H}$ (the bumper is before the house). The same computations as above can be carried out in the interval algebra: the truck being left of the house can be obtained by composing the relationship between the truck and the bumper and the bumper and the house: $\{m\} \times_{13}\{b\}=\{b\}$. In the same way as above, one can enlarge the bumper and the description of the situation will not change until it meets the house, leading to situation (b). This complies with the neighborhood graph of figure $2 b$.

\subsection{Conversion from interval to instant formalisms}

The interval algebra can be obtained directly from the instant algebra through an interval operation (Hirsch 1996) which interprets any element of the interval algebra as a pair of elements of the initial algebra related by a particular relation $(<$ is used for generating $\mathrm{A}_{13}$ from $\mathrm{A}_{3}$ ). It thus deserves the name of interval algebra. The same idea can be used for generating the neighborhoods (Euzenat 1998).

A useful result concerning granularity is that relationships between entities in the interval algebra can be expressed according to the relationships between their bounding instants in the instant algebra. Any relationship between $x=\left\langle x^{-} x^{+}\right\rangle$and $y=\left\langle y^{-} y^{+}\right\rangle$is expressed by a quadruple $\left(r_{1}, r_{2}, r_{3}, r_{4}\right)$ of relationships between the endpoints defined by:

$$
\left\langle x^{-} x^{+>}\left(r_{1}, r_{2}, r_{3}, r_{4}\right)<y^{-} y^{+>} \equiv x^{-} r_{1} y^{-} \wedge x^{-} r_{2} y^{+} \wedge x^{+} r_{3} y^{-} \wedge x^{+} r_{4} y^{+}\right.
$$

assuming that $x<x^{+}$and $y^{-}<y^{+}$, each possible relationship between the bounding instants is expressible by means of such a quadruple. The result for instant/interval algebras is given in table 4 . The symbol $\Rightarrow$ is used so that $\Rightarrow x$ is the expression of an interval as a couple of endpoints and $\Rightarrow r$ a relationship between intervals expressed as a quadruple. $\Rightarrow$ is extended to sets of relations so that $\Rightarrow \rho$ is a set of quadruples.

\begin{tabular}{|c|l|l|l|l||c|l|l|l|l|}
\hline$x \mathrm{ry}$ & $x^{-} \mathrm{r}_{1} y^{-}$ & $x^{-} \mathrm{r}_{2} y^{+}$ & $x^{+} \mathrm{r}_{3} y^{-}$ & $x^{+} \mathrm{r}_{4} y^{+}$ & $x \mathrm{r}^{-1} y$ & $x^{-} \mathrm{r}_{1} y^{-}$ & $x^{-} \mathrm{r}_{2} y^{+}$ & $x^{+} \mathrm{r}_{3} y^{-}$ & $x^{+} \mathrm{r}_{4} y^{+}$ \\
\hline $\mathrm{b}$ & $<$ & $<$ & $<$ & $<$ & $\mathrm{b}^{-1}$ & $>$ & $>$ & $>$ & $>$ \\
\hline $\mathrm{d}$ & $>$ & $<$ & $>$ & $<$ & $\mathrm{d}^{-1}$ & $<$ & $<$ & $>$ & $>$ \\
\hline $\mathrm{O}$ & $<$ & $<$ & $>$ & $<$ & $\mathrm{O}^{-1}$ & $>$ & $<$ & $>$ & $>$ \\
\hline $\mathrm{s}$ & $=$ & $<$ & $>$ & $<$ & $\mathrm{S}^{-1}$ & $=$ & $<$ & $>$ & $>$ \\
\hline $\mathrm{f}$ & $>$ & $<$ & $>$ & $=$ & $\mathrm{f}^{-1}$ & $<$ & $<$ & $>$ & $=$ \\
\hline $\mathrm{m}$ & $<$ & $<$ & $=$ & $<$ & $\mathrm{m}^{-1}$ & $>$ & $=$ & $>$ & $>$ \\
\hline $\mathrm{e}$ & $=$ & $<$ & $>$ & $=$ & $\mathrm{e}$ & $=$ & $<$ & $>$ & $=$ \\
\hline
\end{tabular}

Table 4. The 13 relationships between intervals expressed through relationships between interval endpoints.

Because any formula representing relationship between four instants $x, x^{+}, y^{-}$and $y^{+}$ satisfying the properties of intervals $\left(x<x^{+}\right.$and $\left.y^{-<y^{+}}\right)$can be expressed under that form, the inverse operation $\Leftarrow$ is well-defined. It converts such an expression between bounding instants of two intervals into a set of relations expressing the 
disjunction of relations holding between the intervals. Of course, both operators $(\Leftarrow$ and $\Rightarrow$ ) are inverse.

For instance, one can check that the situation provided as examples for the two previous sections corresponds to the interval algebra. As a matter of fact, the truck (T) being left of $(<)$ the house $(\mathrm{H})$ is described by $\mathrm{T}^{+}<\mathrm{H}^{-}$in the first case and by $\mathrm{T}\{\mathrm{b}\} \mathrm{H}$ in the second one. This corresponds to table 4 because $\mathrm{T}-<\mathrm{T}+<\mathrm{H}^{-}<\mathrm{H}^{+}$(by definition of the intervals) and by using the composition for $\mathrm{A}_{3}$, it can be deduced that $\mathrm{T}^{-}<\mathrm{H}^{-}, \mathrm{T}^{-}<\mathrm{H}^{+}, \mathrm{T}^{+}<\mathrm{H}^{-}$and $\mathrm{T}^{+}<\mathrm{H}^{+}$.

\section{GRANULARITY CONVERSION OPERATORS}

From the above, it can be understood that extended relation algebras constitute a very general way of representing knowledge. The relational representation systems presented so far are adequate for representing a situation at any granularity (as shown in the examples of the previous section).

Meanwhile, the granularity problems presented in the introduction manifest themselves in the context of relation algebra when several representations of the same situation at various granularities have to be compared. As a matter of fact, the situations of figure 1 cannot be merged into one consistent situation: Figures $1 \mathrm{~b}$ and $\mathrm{c}$ together are inconsistent because, in (b), $\mathrm{B}\{\mathrm{m}\} \mathrm{H}$ and, in (c), $\mathrm{B}\{\mathrm{b}\} \mathrm{H}$ which, when paired, i.e. when the set of relationships holding between two objects are intersected, yields $\mathrm{B}\{\} \mathrm{H}$.

The relationships between two representations of the same situation at two different granularities have to be investigated. The present section first presents the notion of operators able to convert a representation from one granularity to another. Then a set of constraints governing these operators is detailed and discussed. This set is made of five core constraints, suitable to any extended relation algebra, and a sixth one dedicated to the construction of interval algebras. In the last sub-section, the problem of guaranteeing the existence of operators satisfying the constraints is explored in a systematic way. The following sections will apply this framework to specific formalisms used for time and space representations.

\subsection{Form of operators}

Operators for transforming the representation of a situation from one granularity to another can be defined which map a representation to a possible set of representations which is compatible with what is observed. The approach taken here adopts the same relational system for representing situations at different granularities. It also assumes that the same set of entities is considered in each representation. As a consequence, only the relationships among entities can vary switching from one granularity to another.

A tool is needed for constraining the modification of the relationships among entities through granularity conversion. It is provided by a couple of functions from $\Gamma$ to $2^{\Gamma}$ called (upward and downward) granularity conversion operators. Given a relationship observed at some particular granularity, the downward (resp. upward) operator provides the set of relationships that can be perceived at a finer (resp. coarser) granularity. These operators are called upward and downward granularity conversion operators and are represented by the infix $\mathrm{g}^{\top} \mathrm{g}^{\prime}$ and $\mathrm{g}^{\prime} \downarrow_{\mathrm{g}}$ operators (where $\mathrm{g}$ and $\mathrm{g}$ ' are granularities such that $\mathrm{g}$ is finer - more precise - than $\mathrm{g}$ '). The operators could have been, like in (Montanari 1996), a binary relationship $\mathrm{g} \leftrightarrow \mathrm{g}$ ' between relations such that $\mathrm{g}_{\mathrm{g}} \leftrightarrow \mathrm{g}^{\prime}\left(r, r^{\prime}\right)$ is equivalent to $r \in{ }_{\mathrm{g}} \uparrow^{\mathrm{g}} r^{\prime}$ and $r^{\prime} \in \mathrm{g}^{\prime} \downarrow_{\mathrm{g}} r$. 
The use of functions emphasizes the transformation aspect and specifies in one expression the set of possible conversions of a particular object. By opposition, the relational views favor the one-to-one relation between two objects. Both forms are equivalent but a functional settings requires a constraint (inverse compatibility) that can be wired in the relational formulation.

A third operator, $g \rightarrow g$, will be used for ${ }_{g} \uparrow g^{\prime}$ and $g^{\prime} \downarrow_{g}$ when the property holds for both (then there is no constraint upon $\mathrm{g}$ and $\mathrm{g}^{\prime}$ ). So, unless stated otherwise, each formula below is universally quantified on the g's, and constrains $g$ to be finer than g' whenever ${ }_{g} \uparrow g^{\prime}$ or $g^{\prime} \downarrow_{g}$ is used. For the sake of completeness, we assume that $g \rightarrow g$ is identity. Last, when it does not matter to identify the granularities (e.g. when asserting abstract properties of the conversion operators), the proposed operators will be replaced by $\uparrow, \downarrow$ or $\rightarrow$.

As usual, the notation $\mathrm{g} \rightarrow \mathrm{g}$, introduced for the conversion of a single relationship, is extended to sets: $\mathrm{g} \rightarrow \mathrm{g}^{\prime} \rho=\bigcup_{r \in \rho} \rightarrow_{g^{\prime}} r$.

These operators can express (and eventually answer to) the three questions asked in the introduction about representations:

- How to convert a representation from one granularity to another one? By applying the operator $\left(r^{\prime}=\mathrm{g} \rightarrow \mathrm{g}^{\prime} r\right)$.

- Are two representations compatible? By applying the operators and testing the compatibility $\left(r^{\prime} \in \mathrm{g} \uparrow^{\mathrm{g}}{ }^{\prime} r\right.$ and $\left.r \in \mathrm{g}^{\prime} \downarrow_{\mathrm{g}} r^{\prime}\right)$.

- What can be the relative granularities of two representations? By testing compatibility in both directions.

The remainder of this section identifies necessary properties of the operators.

\subsection{Properties of granularity conversion operators}

Anyone can think about a particular set of granularity conversion operators by imagining the effects of coarseness. But, here, we identify and discuss a set of properties which should be satisfied by any system of granularity conversion operators. In fact, this set of properties is very small. The next section shows that, in the temporal case, they are sufficient for restricting the number of possible operators to only one (plus the expected operators corresponding to identity and conversion to everything).

\section{Self-conservation}

Self-conservation states that, whatever be the conversion, a relationship must belong to its own conversion. It is quite a sensible and minimal property: the knowledge about the relationship can be less precise but it must have a chance to be correct.

$$
r \in \mathrm{g} \rightarrow \mathrm{g}^{\prime} r \quad \text { (self-conservation) }
$$

Moreover, in a qualitative system, it is possible that nothing changes with granularity if the (quantitative) granularity step is small enough. Not requiring selfconservation would disable the possibility that the same situation looks the same at different granularity. For instance, in figure 1, the conversion from (a) to (c) cannot impose the relation $\mathrm{b}$ (before) between $\mathrm{T}$ and $\mathrm{H}$ to become $\mathrm{m}$ (meets), excluding $\mathrm{b}$, because this would also impose it to (b) in which the relationship has remained the same. 
Self-conservation corresponds to the property named reflexivity when the conversion is a relation (i.e. $\left.\mathrm{g}^{\mathrm{g}} \mathrm{g}^{\prime}(r, r)\right)$. Because (Montanari, 1996) applies to quantitative conversion, it is only true for the conversion which does not change granularity (in fact, reflexivity is universally true, because entities of different granularities belong to disjoint domains).

One might argue that self-conservation is acceptable for upward conversion, but not for downward conversion. However, one-way self conservation plus inverse compatibility (to be discussed below) entails full self-conservation.

Technically, self-conservation ties the conversion to the perceived relation. It disqualifies operators which do not preserve the initial relation (such as the operators which just convert anything to their symmetrical relationships otherwise disqualified by the idempotency property to be discussed below).

\section{Neighborhood compatibility}

A property considered earlier is the order preservation property - stated in (Hobbs 1985) as an equivalence: $\neg(\exists x, y) x>y \wedge \mathrm{g} \rightarrow \mathrm{g}^{\prime} x<\mathrm{g} \rightarrow \mathrm{g}$ ' $y$. Order-preservation takes for granted the availability of an order relation $(<)$ structuring the set of relationships. It states that

$$
\text { if } x>y \text {, then } \neg\left(\mathrm{g} \rightarrow \mathrm{g}^{\prime} x<\mathrm{g} \rightarrow \mathrm{g}, y\right) \quad \text { (order preservation) }
$$

However, it has the shortcoming of requiring the order relation. Its algebraic generalization could be reciprocal avoidance:

$$
\text { if } x \mathrm{r} y \text {, then } \neg\left(\mathrm{g} \rightarrow \mathrm{g}^{\prime} x \mathrm{r}^{-1} \mathrm{~g} \rightarrow \mathrm{g}^{\prime} y\right) \text { (reciprocal avoidance) }
$$

Reciprocal avoidance, is over-generalized and conflicts with self-conservation in case of auto-converse relationships (i.e. such that $r=r^{-1}$ ). The neighborhood compatibility, although not expressed in (Euzenat 1993) has been taken into account informally: it constrains the conversion of a relation to form a conceptual neighborhood (and hence the conversion of a conceptual neighborhood to form a conceptual neighborhood).

] $\forall r . \forall r^{\prime}, r^{\prime} \in \mathrm{g} \rightarrow \mathrm{g}^{\prime} r . \exists r_{1}, \ldots r_{n} \in \mathrm{g} \rightarrow \mathrm{g}^{\prime} r . r_{1}=r^{\prime}, r_{n}=r^{\prime}$, and $\forall i \in[1, n-1] N_{\Gamma}^{X}\left(r_{i}, r_{i+1}\right)$

(neighborhood compatibility)

Neighborhood compatibility has already been reported by Christian Freksa (1992) who considers that a set of relationships must be a conceptual neighborhood in order to be seen as a coarse representation of the actual relationship. [2] is weaker than the two former proposals because it does not prevent the converse to be part of the conversion. But in such a case, it constrains a path between the relation and its converse to be in the conversion too. Neighborhood compatibility seems to be the right property, partly because, unlike the former ones, it does not forbid a very coarse granularity at which any relationship is converted in the whole set of relations. It also seems natural because granularity can hardly be imagined as discontinuous (at least in continuous spaces).

\section{Conversion-reciprocity distributivity}

An obvious property for conversion is symmetry. It states that the conversion of the relation between a first object and a second one must be the converse of the conversion of the relation between the second one and the first one. It is clear that the relationships between two temporal occurrences are symmetrical and thus granularity conversion must satisfy: 


$$
\left(\mathrm{g} \rightarrow \mathrm{g}, \rho^{-1}\right)=\left(\mathrm{g} \rightarrow \mathrm{g}^{\prime} \rho\right)^{-1}
$$

$$
\text { (distributivity of } \mathrm{g} \rightarrow \mathrm{g} \text { over }^{-1} \text { ) }
$$

Failing to implement this property would cause converted representations in which ArB but not $\mathrm{Br}^{-1} \mathrm{~A}$ (e.g. A contains B but B is not contained by A). This is properly in contradiction with the axioms of relation algebras (Tarski 1941).

\section{Inverse compatibility}

Inverse compatibility states that the conversion operators are consistent with each other, i.e. that, if the relationship between two occurrences can be seen as another relationship at some granularity, then the inverse operation from the latter to the former can be achieved through the inverse operator.

$$
r \in \bigcap_{r^{\prime} \in g} \uparrow_{g^{\prime} r}^{g^{\prime}} \downarrow_{g} r^{\prime} \text { and } r \in \bigcap_{r^{\prime} \in \downarrow^{g} \downarrow_{g^{\prime}} r} \uparrow^{g} r^{\prime} \text { (inverse compatibility) }
$$

For instance, if someone in situation (b) of figure 1 is able to imagine that, at a finer granularity (say situation c), there is some space between the bumper and the house, then (s)he must be ready to accept that, if (s)he were in situation (c), (s)he could imagine that there is no space between them at a coarser granularity (as in situation b).

Stated otherwise, inverse compatibility corresponds to the expression $r \in \downarrow r^{\prime}$ iff $r^{\prime} \in \uparrow r$. Inverse compatibility is contained in the relational description of the operator in (Montanari 1996).

\section{Idempotency}

A property which is usually considered first (especially in quantitative systems) is the full transitivity:

$$
\mathrm{g} \rightarrow \mathrm{g} \cdot \mathrm{g}^{\prime} \rightarrow \mathrm{g} " r=\mathrm{g} \rightarrow \mathrm{g}{ }^{\prime} r \quad \text { (transitivity) }
$$

Full transitivity is too strong; it would for instance imply that:

$$
\mathrm{g}^{\top} \mathrm{g}^{\prime} \cdot \mathrm{g}^{\prime} \downarrow_{\mathrm{g}} r=r
$$

Of course, this cannot be achieved because it would mean that there is no loss of information through granularity conversion: this is obviously false. If it were true anyway, there would be no need for granularity operators: everything would be the same at each granularity. On the other hand, the oriented transitivity (previously known as cumulated transitivity) can be expected:

$$
\mathrm{g} \uparrow \mathrm{g} \cdot{ }_{\mathrm{g}} \uparrow \mathrm{g} " r={ }_{\mathrm{g}} \uparrow \mathrm{g} " r \text { and } \mathrm{g} " \downarrow_{\mathrm{g}}, \mathrm{g} \text { ' } \downarrow_{\mathrm{g}} r=\mathrm{g} " \downarrow_{\mathrm{g}} r \text { (oriented transitivity) }
$$

However, in a purely qualitative calculus, the actual granularities g, g' and g" are not relevant and oriented transitivity becomes a property of idempotency of operators:

$$
\uparrow \cdot \uparrow=\uparrow \text { and } \downarrow \cdot \downarrow=\downarrow
$$

\section{(idempotency)}

At first sight, it could be clever to have non-idempotent operators which are less and less precise with granularity conversion. However, if this applies very well to quantitative data, it does not apply for qualitative: the qualitative conversion applies equally for a large granularity conversion and for a small one which is ten times less. If, for instance, in a particular situation, a relationship between two entities is $r$, in a coarser representation it is $r$ ' and in an even coarser representation it is $r$ ", then $r$ " must be a member of the upward conversion of $r$. This is because $r$ " is indeed the 
result of a qualitative conversion from the first representation to the third. In figure 1 , for instance, if the black band on the truck can be equal (longitudinally, e) to the truck body (a), then can start (s) the truck body (b) and then be contained (d) in the truck body (c). Obviously, the downward conversion of e must contain s and $d$ because the conversion from (a) to (c) is also a downward conversion of e. Thus, qualitatively, $\downarrow \cdot \downarrow=\downarrow$.

If there were no idempotency, converting a relationship directly would give a different result than when doing it through ten successive conversions.

\section{Representation independence}

The core properties given so far were not related to the interval relation algebras. This kind of algebra can be constrained so that the conversion operators of both algebras are connected. The property constraining the conversion operators is based on the operation that allows one to switch from a relation algebra to an interval relation algebra (by $\Leftarrow$ and $\Rightarrow$ ). Representation independence states that the conversion must not be dependent upon the representation of the temporal entity (as an interval or as a set of bounding instants):

$$
\begin{aligned}
& \mathrm{g} \rightarrow \mathrm{g}^{\prime} \rho=\Leftarrow \mathrm{g} \rightarrow \mathrm{g}^{\prime} \Rightarrow \rho \\
& \text { and (representation independence) } \\
& \mathrm{g} \rightarrow \mathrm{g}^{\prime} \rho=\Rightarrow \mathrm{g} \rightarrow \mathrm{g}^{\prime} \Leftarrow \rho
\end{aligned}
$$

An example of representation independence, that refers to figure 1, will be given in the next section in which the instant algebra and the interval algebra are used for expressing the same situations and yield the same result.

Note that, because $\Leftarrow$ requires that the relationship between bounding instants are that of an interval, the results are restricted to those which can be interpreted as relations between valid intervals. The extra relations correspond to the vanishing of an interval, or the transformation of an interval into a point, which are not considered here.

Representation independence can be formulated as distributivity:

$$
\Rightarrow\left(\mathrm{g} \rightarrow \mathrm{g}^{\prime} \rho\right)=\mathrm{g} \rightarrow \mathrm{g}^{\prime}(\Rightarrow \rho) \text { and } \Leftarrow\left(\mathrm{g} \rightarrow \mathrm{g}^{\prime} \rho\right)=\mathrm{g} \rightarrow \mathrm{g}^{\prime}(\Leftarrow \rho)
$$

DEFINITION 1: Given an extended relation algebra, a couple of operators up-down satisfying properties [1] through [5] (core properties) is a coherent granularity conversion operator for that system.

The framework provided so far concerns two operators related by the constraints but there is no specificity of the upward or downward operator (this is why constraints are symmetrical). By convention, if the system contains a unique equivalence relation (defined as e such that $\mathrm{e}=\mathrm{eoe}=\mathrm{e}^{-1}$ (Hirsch 1996)), the operator which maps the equivalence relation to a strictly broader set is denoted as the downward operator (if unique). This meets the intuition because the coarser the view the more indistinguishable the entities (and they are then subject to the equivalence relation). Implementing this requirement as another constraint would have two consequences:

- simplifying the treatment below;

- allowing the introduction of conversion-specific constraints.

However, this is not necessary for the work presented here, so the more general solution, which does not introduce orientation, has been retained. 


\subsection{Existence results for extended relation algebras}

For any extended relation algebra there are two operators which always satisfy these requirements: the identity function (Id) which maps any relation into itself (or a singleton containing itself) and the non-informative function (NI) which maps any relation into the base set of the algebra. It is noteworthy that these functions must then be their own inverse (i.e. they are candidates for both $\uparrow$ and $\downarrow$ at once). These solutions are not considered anymore below. The question of the general existence of granularity conversion operators, different from Id and NI, corresponding to the above constraints can be raised. Two partial results are established. The first one shows that there are small algebras with no non-trivial operators:

PROPOSITION 1: The extended relation algebra based on two distinct elements a and $\mathrm{a}^{-1}$ such that $N\left(\mathrm{a}, \mathrm{a}^{-1}\right)$ has no granularity conversion operators other than identity and non-informative map.

It can be noted that in the case of two auto-inverse operators (e.g., $=$ and $\neq$ ), there must exist conversion operators as shown by proposition 2. Proposition 2 exhibits a systematic way of generating operators from minimal requirements (but does not provide a way to generate all the operators). It only provides a sufficient but not necessary condition for having operators.

PROPOSITION 2: Given an extended relation algebra containing at least two relationships $\mathrm{a}$ and $\mathrm{b}$ such that neighborhood is the total relation on the relationships, there exists a couple of upward/downward granularity operators defined by:

if $\mathrm{a}$ and $\mathrm{b}$ are auto-inverse: $\downarrow \mathrm{a}=\{\mathrm{a}, \mathrm{b}\}, \uparrow \mathrm{b}=\{\mathrm{a}, \mathrm{b}\}$ the remainder being identity;

if a only is auto-inverse: $\downarrow a \mathrm{a}=\left\{\mathrm{a}, \mathrm{b}, \mathrm{b}^{-1}\right\}, \uparrow \mathrm{b}=\{\mathrm{a}, \mathrm{b}\}, \uparrow^{-1}=\left\{\mathrm{a}, \mathrm{b}^{-1}\right\}$, the remainder being identity;

if $\mathrm{a}$ and $\mathrm{b}$ are not auto-inverse: $\downarrow_{\mathrm{a}}=\{\mathrm{a}, \mathrm{b}\}, \uparrow \mathrm{b}=\{\mathrm{a}, \mathrm{b}\}, \downarrow^{-1}=\left\{\mathrm{a}^{-1}, \mathrm{~b}^{-1}\right\}, \uparrow^{-1}=\left\{\mathrm{a}^{-1}\right.$, $\left.\mathrm{b}^{-1}\right\}$, the remainder being identity.

There can be, in general, many possible operators for a given algebra. Proposition 2 shows that the five core properties of $\$ 3.2$ are consistent. Another general question about them concerns their independence. It can be answered affirmatively.

PROPOSITION 3: The core properties of granularity operators are independent.

This is proven by providing five systems satisfying all properties but one.

\section{APPlication to temporal algebras}

This general framework for granularity conversions in extended relation algebras is here applied to specific temporal systems. The theory is first applied to the instant algebra against which the constraints are eliminated in order to generate the only non-trivial couple of operators. Transferring these results to the well-known interval algebra is straightforward thanks to the sixth constraint which builds the operators through the interval construction. The last results in this section concern the distributivity of granularity conversion over relation composition. 


\subsection{Conversion operators for the instant algebra}

Once the properties have been defined, one can start generating candidate upward and downward conversion operators. However, the requirements are so precise that they leave no place for choice. It is shown below, by starting with the instant algebra, that there is only one possible couple of operators.

Table 5 features all the $64\left(=2^{3} .2^{3}\right)$ imaginable conversion operators for the instant algebra. Each cell identifies an operator: the column determines the conversion of "=" and the row determines that of " $<"$. The conversion of " $>$ " is fully determined by that of "<" (through property [3]).

\begin{tabular}{|c|c|c|c|c|c|c|c|c|}
\hline$\langle\backslash=$ & \{\} & $\{<\}$ & $\{=\}$ & $\{>\}$ & $\{<=\}$ & $\{\langle>\}$ & $\{=>\}$ & $\{\langle\Leftrightarrow\}$ \\
\hline\{\} & $1 \backslash 1$ & $1 \backslash 1$ & 11 & $1 \backslash 1$ & $1 \backslash 3$ & $1 \backslash 1,2$ & $1 \backslash 3$ & 11 \\
\hline$\{<\}$ & $\backslash 1$ & $\backslash 1$ & Id & $\backslash 1$ & $\backslash 3$ & $\backslash 1,2$ & $\backslash 2,3$ & $\alpha$ \\
\hline$\{=\}$ & $1 \backslash 1$ & $1 \backslash 1$ & 11 & $1 \backslash 1$ & $1 \backslash 3$ & $1 \backslash 1,2$ & $1 \backslash 3$ & 11 \\
\hline$\{>\}$ & $1 \backslash 1$ & $1 \backslash 1$ & $1 \backslash$ & $1 \backslash 1$ & $1 \backslash 3$ & $1 \backslash 1,2$ & $1 \backslash 3$ & $1 \backslash$ \\
\hline$\{<=\}$ & $\backslash 1$ & $\backslash 1$ & $\beta$ & $\backslash 1$ & $\backslash 3$ & $\backslash 1,2$ & $\backslash 3$ & $\gamma$ \\
\hline$\{<>\}$ & $2 \backslash 1$ & $2 \backslash 1$ & $2 \backslash$ & $2 \backslash 1$ & $2 \backslash 3$ & $2 \backslash 1,2$ & $2 \backslash 3$ & 21 \\
\hline$\{=>\}$ & $1 \backslash 1$ & $1 \backslash 1$ & $1 \backslash$ & $1 \backslash 1$ & $1 \backslash 3$ & $1 \backslash 1,2$ & $1 \backslash 3$ & $1 \backslash$ \\
\hline$\{<=>\}$ & $\backslash 1$ & $\backslash 1$ & $\delta$ & $\backslash 1$ & $\backslash 3$ & $\backslash 1,2$ & $\backslash 3$ & $\begin{array}{l}\text { no } \\
\text { info }\end{array}$ \\
\hline
\end{tabular}

Table 5. Each column represents a possible conversion for $=$ and each row represents a possible conversions for $<$. Because the conversion for > is constrained by [3] to be the converse of that of <, it is not considered here. Thus, each cell represents a conversion operator (defined on the base relations). It contains the numbers corresponding to constraints violated by the corresponding operator (before the backslash, those violated by <; after it, those violated by the conversion of $=$ ).

These a priori possible operators for converting $<$ and $=$ can be easily reduced to six: Constraint [1] restricts the conversion of $<$ to be $\{<\},\{<=\},\{<>\}$ or $\{<=>\}$ and that of $\{=\}$ to be in $\{=\},\{<=\},\{=>\}$ or $\{<=>\}$. Constraint [2] suppresses the possibility for $<$ to become $\{<>\}$. Constraint $[3]$ has been used in a peculiar but correct way for eliminating the $\{<=\}$ (resp. $\{=>\}$ ) solutions for $=$. As a matter of fact, these solutions would cause the conversion of $=^{-1}$ to be $\{=>\}$ (resp. $\{<=\}$ ), but $=^{-1}$ is $=$ and thus its conversion should be that of $=$ too.

\begin{tabular}{l|ll}
$<\backslash=$ & $\{=\}$ & $\{<=>\}$ \\
\hline$\{<\}$ & Id & $\alpha$ \\
$\{<=\}$ & $\beta$ & $\gamma$ \\
$\{<=>$ & $\delta$ & no info
\end{tabular}

Table 6. The six possible conversion operators for $=$ and $<$.

There are still six possible conversion operators left (Id, $\alpha, \beta, \gamma, \delta$ and NI). The above table does not consider whether the operators are for downward or upward conversion. This leaves, a priori, 36 upward-downward couples. But the use of property [4] — the putative operators must be compatible with their inverse operator (and vice-versa) — reduces them to 3: Id-Id, $\alpha-\beta$ and NI-NI.

\begin{tabular}{c|cccccc}
. accepts & $\operatorname{Id}$ & $\alpha$ & $\beta$ & $\gamma$ & $\delta$ & NI \\
\hline. & & & & & & \\
\hline$\alpha$ & - & $\bullet$ & $\bullet$ & $\bullet$ & $\bullet$ & $\bullet$ \\
$\beta$ & - & $\bullet$ & $\bullet$ & $\bullet$ & $\bullet$ & $\bullet$ \\
$\gamma$ & - & - & - & - & - & $\bullet$ \\
$\delta$ & - & - & - & - & - & $\bullet$
\end{tabular}


Table 7. From the 6 possible operators satisfying individual properties [1], [2] and [3], 36 couples of upward/downward operators can be considered. Among them only the 18 cells marked with an $\bullet$ are compatible with the first equation of property [4]. But, because the compatibility must also be true in the reverse order, only the couples compatible in both orders remain.

The solution Id-Id cannot be considered as granularity because it does not provide any change in the representation. The solution NI-NI is useless. Thus the $\alpha-$ $\beta$ pair is chosen as downward/upward operators (according to the convention provided above).

PROPOSITION 4: The table 8 defines the only possible non-auto-inverse upward/downward operators for $\mathrm{A}_{3}$.

\begin{tabular}{lll} 
relation: $\mathrm{r}$ & $\mathrm{g}^{\uparrow \mathrm{g}^{\prime} \mathrm{r}}$ & $\mathrm{g}_{\mathrm{g}}, \mathrm{r}$ \\
\hline$<$ & $<=$ & $<$ \\
$=$ & $=$ & $<=>$ \\
$>$ & $>=$ & $>$
\end{tabular}

Table 8. Upward and downward granularity conversions between instants.

The $\alpha-\beta$ couple fits intuition very well. For instance, if the example of figure 1 is modeled through bounding instants ( $x^{-}$for the left endpoint and $x^{+}$for the right endpoint) of intervals $\mathrm{T}^{+}, \mathrm{B}^{-}, \mathrm{B}^{+}$and $\mathrm{H}^{-}$, it is represented in (c) by $\mathrm{T}^{+}=\mathrm{B}^{-}$(the truck ends where the bumper begins), $\mathrm{B}^{-}<\mathrm{B}^{+}$(the beginning of the bumper is before its end), $\mathrm{B}^{+}<\mathrm{H}^{-}$(the end of the bumper is before the beginning of the house) in (b) by $\mathrm{B}^{+}=\mathrm{H}^{-}$(the bumper ends where the house begins) and in (a) by $\mathrm{B}^{-}=\mathrm{B}^{+}$(the bumper does not exist anymore). This is possible by using the couple $\alpha-\beta$ which converts $\mathrm{B}^{+}<\mathrm{H}^{-}$into $\mathrm{B}^{+}=\mathrm{H}^{-}(=\in \beta<)$ and $\mathrm{B}^{-}=\mathrm{B}^{+}$into $\mathrm{B}^{-}<\mathrm{B}^{+}(<\in \alpha=)$, but not with the use of $\gamma$ as a downward operator.

The operators of table 8 also satisfy the properties of granularity operators.

PROPOSITION 5: The upward/downward operators for $A_{3}$ of table 8 satisfy the properties [1] through [5].

\subsection{Granularity operators for the interval algebra}

Many operators for the interval algebra can be generated by enumeration. But it must be related to the instant algebra by constraint [6]. Table 9 shows the automatic translation from points to intervals generated by this constraint:

\begin{tabular}{|c|c|c|c|c|c|c|c|c|c|c|}
\hline$r$ & \multicolumn{4}{|c|}{$\uparrow \Rightarrow r$} & $\mathrm{~g}{ }^{\uparrow} \mathrm{g}^{\prime} r$ & \multicolumn{4}{|c|}{$\downarrow \Rightarrow r$} & $\mathrm{~g} \downarrow_{\mathrm{g}}, r$ \\
\hline $\mathrm{b}$ & $<=$ & $<=$ & $<=$ & $<=$ & b m & $<$ & $<$ & $<$ & $<$ & $\mathrm{b}$ \\
\hline $\mathrm{d}$ & $>=$ & $<=$ & $>=$ & $<=$ & $\mathrm{ds} f \mathrm{e}$ & $>$ & $<$ & $>$ & $<$ & d \\
\hline o & $<=$ & $<=$ & $>=$ & $<=$ & os me $f^{-1}$ & $<$ & $<$ & $>$ & $<$ & o \\
\hline S & $=$ & $<=$ & $>=$ & $=$ & $\mathrm{se}$ & $\Leftrightarrow$ & $<$ & $>$ & $<$ & o s d \\
\hline$f$ & $>=$ & $<=$ & $>=$ & $=$ & $\mathrm{fe}$ & $>$ & $<$ & $>$ & $\Leftrightarrow$ & $\mathrm{o}^{-1} \mathrm{fd}$ \\
\hline $\mathrm{m}$ & $<=$ & $<=$ & $=$ & $<=$ & $\mathrm{m}$ & & $<$ & $\Leftrightarrow$ & $<$ & b m o \\
\hline e & $=$ & $<=$ & $>=$ & $=$ & e & $\Leftrightarrow$ & $<$ & $>$ & $<=>$ & $\begin{array}{l}o f^{-1} d^{-1} \text { s e s }{ }^{-1} \\
d f^{-1} o^{-1}\end{array}$ \\
\hline
\end{tabular}


Table 9. Transformations of upward and downward operators between instants into interval relation quadruples.

The constraint that it must be the interval algebra (in the sense of Hirsch (1996), see $\$ 2.4$ ) of the instant algebra restricts the extraction of interval relations to the valid ones. Table 10 provides the only possible operators for $\mathrm{A}_{13}$.

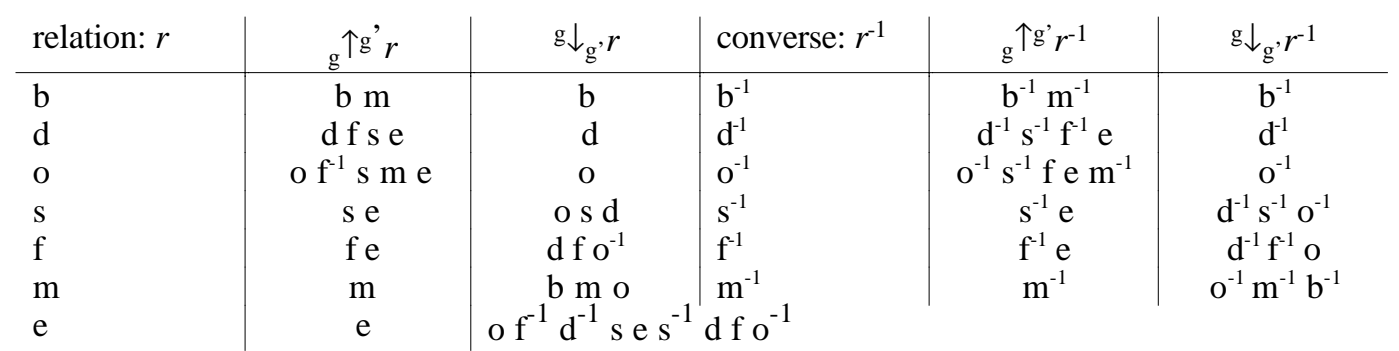

Table 10. Upward and downward conversion operators between intervals.

PROPOSITION 6: The upward/downward operators for $\mathrm{A}_{13}$ of table 10 are the only ones which satisfy the property [6] with regard to the operators for $A_{3}$ of table 8 .

The corresponding operators enjoy the same properties as the operators for $A_{3}$.

PROPOSITION 7: The upward/downward operators for $\mathrm{A}_{13}$ of table 10 satisfy the properties [1] through [5].

The reader is invited to check on the example of figure 1, that what has been said about instant operators is still valid: the situation (c) is described by $T\{m\} B$ (the truck meets its bumper), $\mathrm{B}\{\mathrm{b}\} \mathrm{H}$ (the bumper is aside the house), in (b) by $\mathrm{B}\{\mathrm{m}\} \mathrm{H}$ (the bumper meets the house) and in (c) where the bumper does not appear anymore by $\mathrm{T}\{\mathrm{m}\} \mathrm{H}$ (the truck meets the house). This is compatible with the idea that, at a coarser granularity, $b$ can become $m(m \in \uparrow b)$ and that, at a finer granularity, $m$ can become $b(b \in \downarrow m)$.

The identified upward operator does not satisfy the constraint [2] for Bneighborhood (in which intervals are translated continuously), as it is violated by d, $\mathrm{s}$ and $\mathrm{f}$, and $\mathrm{C}$-neighborhood (in which intervals are continuously expanded or contracted while preserving their center of gravity - i.e. the bounds are translated in opposite directions of the same quantity) as it is violated by $\mathrm{o}, \mathrm{s}$ and $\mathrm{f}$. Constraint [2] is violated because the corresponding neighborhoods are not based upon independent limit translations although this independence has been used for translating the results from $\mathrm{A}_{3}$ to $\mathrm{A}_{13}$.

The identified operators correspond exactly to the closure of relationships that Gérard Ligozat (1990) introduced in his formalism (see Figure 3). This is natural because the closure, just like the conversion operators, provides all the adjacent relationships of a strictly higher dimension (here the dimension of a relationship depends on the number of endpoints it constrains, i.e. the number of "=" relation in the rows of table 4 ).

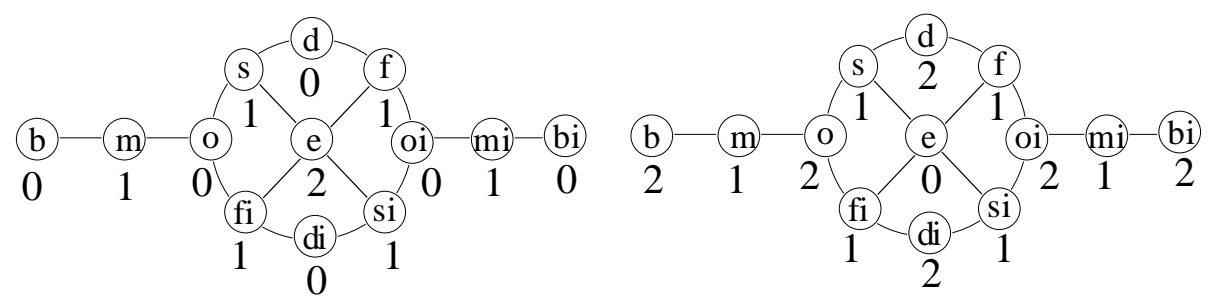


Figure 3. Transposition of Ligozat (1990) framework on the A-neighborhood graph. Each node is labeled with the number of endpoints that its relationship constrains (resp. does not constrain) when one of the intervals is given. The upward (resp. downward) conversion operator is obtained by gathering, for each relation, the reachable nodes by following a path with strictly ascending labels.

\subsection{Granularity and composition}

The composition of symbolic relationship is a favored inference means for symbolic representation systems. One of the properties which would be interesting to obtain is the independence of the results of the inferences from the granularity level [7] which is denoted by the distributivity of $\rightarrow$ over $\times$.

$$
\rightarrow\left(\rho_{1} \times \rho_{2}\right)=\left(\rightarrow \rho_{1}\right) \times\left(\rightarrow \rho_{2}\right)
$$

$$
\text { (distributivity of } \rightarrow \text { over } \times \text { ) }
$$

This property is only satisfied for upward conversion in $\mathrm{A}_{3}$.

PROPOSITION 8: The upward operator for $\mathrm{A}_{3}$ satisfies property [7].

It does not hold true for $\mathrm{A}_{13}$ : let $x, y$ and $z$ be three intervals such that $x \mathrm{~b} y$ and $y \mathrm{~d} z$, the application of composition of relations gives $x\{\mathrm{~b} 0 \mathrm{~m} \mathrm{~d} \mathrm{~s}\} z$ which, once upwardly converted, yields $x\left\{\mathrm{~b} \mathrm{me} \mathrm{d} \mathrm{f} \mathrm{s} \mathrm{o}{ }^{-1}\right\} z$. On the contrary, if the conversion is first applied, it returns $x\{\mathrm{~b} \mathrm{~m}\} y$ and $y\{\mathrm{~d} \mathrm{f} \mathrm{s} \mathrm{e}\} z$ which, once composed, gives $x\{\mathrm{~b}$ o m $\mathrm{d} s\} z$. The interpretation of this result is the following: by first converting, the information that there exists an interval $y$, forbidding $x$ to finish $z$, is lost: if, however, the relationships linking $y$ to $x$ and $z$ are kept, then the propagation will take them into account and recover the lost precision: $\left\{\mathrm{b} \mathrm{m} \mathrm{e} \mathrm{d} \mathrm{s} \mathrm{o} \mathrm{f}^{-1}\right\} \wedge\{\mathrm{b} \circ \mathrm{m} \mathrm{ds}\}=\{\mathrm{b} \circ \mathrm{md}$ s\}. However, this cannot be prevented because, if the length of $y$ is so small that the conversion makes it vanish, the correct information at that granularity is the one provided by applying first the composition: $x$ can meet $z$ at such a granularity.

Although [7] cannot be achieved for upward conversion in $A_{13}$, upward conversion is super-distributive over composition.

PROPOSITION 9: The upward operator for $\mathrm{A}_{13}$ satisfies the following property:

$$
\left(\uparrow \rho_{1}\right) \times\left(\uparrow \rho_{2}\right) \subseteq \uparrow\left(\rho_{1} \times \rho_{2}\right) \quad \text { (super-distributivity of } \uparrow \text { over } \times \text { ) }
$$

A similar phenomenon appears with the downward conversion operators (it appears both for instants and intervals). So let $x, y$ and $z$ be three instants such that $x>y$ and $y=z$. On one hand, the composition of relations gives $x>z$, which is converted to $x>z$ at the finer granularity. On the other hand, the conversion yields $x>y$ and $y\{\langle>\} z$ because, at a more precise granularity $y$ could be close but not really equal to $z$. The composition then provides no more information about the relationship between $x$ and $z(x\{\langle=>\} z)$. This is the reverse situation as before: it takes into account the fact that the non-distinguishability of two instants cannot be ensured at a finer granularity. Of course, if everything is converted first, then the result is as precise as possible: downward conversion is sub-distributive over composition.

PROPOSITION 10: The downward operators for $A_{13}$ and $A_{3}$ satisfy the following property: 


$$
\begin{aligned}
\downarrow & \left(\rho_{1} \times \rho_{2}\right) \subseteq\left(\downarrow \rho_{1}\right) \times\left(\downarrow \rho_{2}\right) \\
& (\text { sub-distributivity of } \downarrow \text { over } \times)
\end{aligned}
$$

The two latter properties can be useful for propagating constraints in order to obtain the maximum of information quickly. For instance, in the case of upward conversion, if no interval vanishes, every relationship must be first converted and then composed.

These properties have been discovered independently in the quantitative granularity setting through an approximation algorithm for quantitative constraints (Bettini 1998a).

\subsection{Discussion: interpretation of granularity conversion}

Time is usually interpreted as a straight line, instants as points and intervals as segments. Under a numerical light, granularity can be defined as scaling plus filtering what is relevant and what is not (a discretization). However, granularity is a filter on size as the name indicates. For the case of time, the granularity of a system can be defined as the duration of the smallest relevant event (relevance being defined independently beforehand). This applies obviously to intervals and less obviously to points where it is the shortest period that must occur between two instants so that they are considered as different.

This has consequences for symbolic representations: if, at a coarse granularity, one observes that some event is connected to another this can be wrong at a finer granularity because an irrelevant lapse of time could be relevant there. In another way, when communicating the same observation, short lapses of time may be irrelevant (and thus the relationship between the event can be disconnected). It is what happened for the relationship between $\mathrm{B}$ and $\mathrm{H}$, which is $\{\mathrm{b}\}$, in Figure $1 \mathrm{c}$, and becomes $\{\mathrm{m}\}$, in $1 \mathrm{~b}$.

The present study focuses on objects which persist and keep the same nature (i.e. an interval persists as an interval) from one granularity to another. This is because only the relationships between these objets have been considered and when objects vanish they hold no relation with anything. As a matter of fact, the vanishing of $B$ in Figure 1a has not been accounted for by any conversion of the relation between $\mathrm{B}$ and something else.

There are open issues not considered here. One is the establishment of operators for an extended relation algebra of both points and intervals in which an interval can become a point (and vice versa) through continuous transformation. Another one is the explicit consideration of the vanishing objects.

\section{APPLiCATION TO TWO SPACE REPRESENTATIONS}

Another possible application of algebras of relations is space. Intuitively, granularity applies to space and there must be operators for qualitative representations of space. It is thus interesting to explore space granularity.

Qualitative time representation has inspired several extensions towards qualitative space representation (see (Hernández 1994) for a summary). However, there is not a universally accepted representation. There are several proposed representations for space (Güsgen 1989; Egenhofer\& 1992; Randell\& 1992a) which can be considered as starting points for space representation and not as full-fledged representation systems.

Instead of considering specific formalisms, we try to transfer the results found above to classes of systems obtained by certain constructions. Below two 
constructors are introduced which transform one extended relation algebra into another. They are Cartesian product (\$5.1) and weakening (\$5.2). These constructors have already been used for generating space representations. So, they are ideal candidate. The constructors are proven to preserve several properties of the granularity conversion operators.

\subsection{Product of the interval algebra}

The classical model of the interval algebra is the mono-dimensional Euclidean space. It can be easily extended to an $n$-dimensional Euclidean space through Cartesian product (Güsgen 1989). In such an extension, it is straightforward to define instants as points and areas as intervals. An interval is an area delimited by two points, i.e. a (hyper-)parallelogram whose borders are parallel to the axes of the reference frame. The extension of the time algebra is defined as follows. From a structure $\left\langle\Gamma,{ }^{-1}, \times, N\right\rangle$, another structure $\left\langle\Gamma^{n}, \times^{n},{ }^{-1}, N^{n}\right\rangle$ is defined such that:

$\forall r_{1} \in \Gamma, \ldots r_{n} \in \Gamma, r=\left(r_{1}, \ldots r_{n}\right) \in \Gamma^{n}$ (also noted by $r=\underset{i=1}{\times} r_{i}$ ). This notation is extended to sets: $\underset{i=1}{\stackrel{n}{\times}} \rho_{i}=\left\{\underset{i=1}{\stackrel{n}{\times}} r_{i} / r_{i} \in \rho_{i}\right\}$.

$\left({ }^{-1}\right.$ definition on $\left.\Gamma^{n}\right)$

$$
r^{-1}=\underset{i=1}{n} r_{i}^{-1}=\left(r_{1}^{-1}, \ldots r_{n}^{-1}\right)
$$

$\left(\times\right.$ definition on $\left.\Gamma^{n}\right) \quad r \times r^{\prime}=\underset{i=1}{\times} r_{i} \times r_{i}^{\prime}=\underset{r_{1}^{\prime \prime} \in r_{1} \times r_{1}^{\prime}, \ldots r_{n}^{\prime \prime} \in r_{n} \times r_{n}^{\prime}}{\bigcup}\left\{\left(r_{1}^{\prime \prime}, \ldots r_{n}^{\prime \prime}\right)\right\}$

$\left(N\right.$ definition on $\left.\Gamma^{n}\right) \quad N(r)=\stackrel{n}{\times} N\left(r_{i}\right)=\underset{r_{1}^{\prime \prime} \in N\left(r_{1}\right), \ldots r_{n}^{\prime \prime} \in N\left(r_{n}\right)}{\bigcup}\left\{\left(r_{1}^{\prime \prime}, \ldots r_{n}^{\prime \prime}\right)\right\}$

The extension can be applied to granularity conversion operators:

$$
\left(\rightarrow \text { definition on } \Gamma^{n}\right) \rightarrow r=\underset{i=1}{\times} \rightarrow r_{i}=\underset{r_{1}^{\prime} \in \rightarrow r_{1}, \ldots . r_{n}^{\prime} \in \rightarrow r_{n}}{\bigcup}\left\{\left(r^{\prime}, \ldots r_{n}^{\prime}\right)\right\}
$$

These operators are extended to sets of relations in such a way that

$$
\rho^{-1}=\left\{r^{-1} \mid r \in \rho\right\}, \rho \times \rho^{\prime}=\bigcup_{r \in \rho, r^{\prime} \in \rho^{\prime}} r \times r^{\prime} \text { and } \rightarrow \rho=\bigcup_{r \in \rho} \rightarrow r .
$$

This is sufficient for defining the conversion operators in $n$-dimensional spaces. First, the properties of granularity operators are preserved.

PROPOSITION 11: If the upward/downward operators for $\Gamma$ satisfy the properties [1] through [5], then the upward/downward operators for $\Gamma^{n}$ satisfy them too.

The preservation of representation independence depends on the existence of operators for converting from one representation into another. The operators are defined here entirely through the product notation (so quadruples are products of four dimensions). Although the notation is convenient one, should keep in mind that these quadruples do not represent the relationships between the same objects and 
that additional constraints must be satisfied by the quadruples. This corresponds to the expression of the bounds of $n$-dimensional intervals.

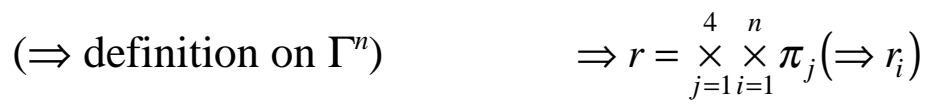

in which $\pi_{k}$ is a notation satisfying the following equation:

$\left(\pi_{k}\right.$ definition $) \quad \pi_{k}\left(\begin{array}{c}\underset{m}{x} r_{i} \\ i=1\end{array}\right)=r_{k} \quad$ with $1 \leq \mathrm{k} \leq \mathrm{m}$

$\left(\Leftarrow\right.$ definition on $\left.\Gamma^{n}\right) \quad \Leftarrow \underset{j=1}{\times} \underset{i=1}{\times} r_{i}^{j}=\stackrel{n}{\underset{i=1}{\times}} \underset{i}{\stackrel{4}{\times}} r_{i}^{j}$

when defined.

PROPOSITION 12: Let $\mathrm{H}$ be an algebra of relations and $\Gamma$ one of its interval algebras, if the upward/downward operators for $\Gamma$ satisfy the property [6] with regard to the operators of $\mathrm{H}$, then the upward/downward operators for $\Gamma^{n}$ satisfy the property [6] with regard to the operators of $\mathrm{H}^{n}$.

Finally, the product construction preserves the distributivity results obtained for the base structure.

PROPOSITION 13: The upward/downward operators for $\Gamma^{n}$ satisfy the same properties as $\Gamma$ with regard to properties [7], [8] and [9].

\subsection{Weakening of relation algebras}

The weakening of a relation algebra will show less details in the characterization of the relationship. A weakened algebra is obtained by grouping several relationships together (it is a quotient). Weakening $\left(\Downarrow: 2^{\Gamma} \rightarrow 2^{\Gamma^{\prime}}\right)$ and strengthening $\left(\Uparrow: \Gamma^{\prime} \rightarrow 2^{\Gamma}\right)$ constructors from an initial algebra $\Gamma$ to a weak algebra $\Gamma^{\prime}$ are thus introduced. Given an existing algebra $\Gamma$ and the weakened algebra $\Gamma^{\prime}$ such that $\Uparrow$ transforms a $\Gamma^{\prime}$-relationship into a set of $\Gamma$-relationships and the image of $\Gamma^{\prime}$ by $\Uparrow$ is a partition of $\Gamma$ (i.e. a set of disjoint non-empty subsets of $\Gamma$ covering $\Gamma$ ), $\Downarrow$ can be defined by the function associating to any $\Gamma$-relationship the $\Gamma$ '-relationship whose image by $\Uparrow$ contains it. These constructors are extended to the sets of relationships as usual. They satisfy the following property:

[w1]

$$
\Downarrow \Uparrow \rho=\rho
$$

If the considered set $\rho$ is either the result of a $\Uparrow$ transformation or the union of such sets, the property [w2] also holds:

[w2]

$\Uparrow \Downarrow_{\rho=\rho}$

Otherwise, the following weaker property holds:

[w3]

$$
\rho \subseteq \Uparrow \Downarrow_{\rho}
$$

These transformation are closely related to the concept of qualitative constraint abstraction introduced in (Güsgen 1989) and they can be defined accordingly, but instead of being used from a quantitative representation to a qualitative 
representation, they are used between two qualitative representations. Converse, composition and neighborhood are defined through weakening:

$\left({ }^{-1}\right.$ definition on $\left.\Gamma^{\prime}\right)$

$\left(\times\right.$ definition on $\left.\Gamma^{\prime}\right)$

$\left(N\right.$ definition on $\left.\Gamma^{\prime}\right)$

$$
\begin{gathered}
\rho^{-1}=\Downarrow(\Uparrow \rho)^{-1} \\
\rho_{1} \times \rho_{2}=\Downarrow\left(\Uparrow \rho_{1} \times \Uparrow \rho_{2}\right) \\
N_{\Gamma^{\prime}}\left(r^{\prime}\right)=\Downarrow \bigcup_{r \in \Uparrow r^{\prime}} N_{\Gamma}(r)
\end{gathered}
$$

These definitions can be applied to $\mathrm{A}_{3}$ and $\mathrm{A}_{13}$ yielding the relationships considered in table 11 and 12 . It must be noted that the resulting relationship looks like sets of qualitative relationships well known in the spatial temporal representation: those of the point algebra in space and the Region Connection Calculus (or RCC-8) algebra (Randell\& 1992a).

These definitions do not indeed yield the actual RCC-8 algebra because the weakening process has not forgotten that the considered objects are intervals, and thus, the composition is not that of RCC-8 (the demonstration is out of scope here). However, the weakening provides a set of relationships that can be put in bijection with those of RCC-8 and, as will be shown, it also provides a couple of valid (in the sense proposed in previous sections) granularity conversion operators for RCC-8.

\begin{tabular}{lll} 
relation: $r$ & ${ }^{\uparrow}{ }^{\prime}{ }^{\prime} r$ & ${ }^{\downarrow} \downarrow \mathrm{g}^{\prime} r$ \\
\hline$=$ & $=$ & $=\neq$ \\
$\neq$ & $\neq=$ & $\neq$
\end{tabular}

Table 11. Conversion operators for topological spatial relationship between points $\left(\mathrm{A}_{2}\right)$.

$\begin{array}{lll}\text { relation }(r): x 1 r \times 2 & { }_{\mathrm{g}}{ }^{\downarrow \mathrm{g}^{\prime}} r \\ \text { nto (non-tangential outside) } & \text { nto }\end{array}$

Table 12. Conversion operators for topological spatial relationship between areas ( $\left.\mathrm{A}_{8}\right)$.

The weakened (upward and downward) conversion operators can be defined accordingly:

$\left(\rightarrow\right.$ definition on $\left.\Gamma^{\prime}\right) \quad \rightarrow \rho=\Downarrow \rightarrow \Uparrow \rho$

It is not claimed that weakening provides the only possible upward-downward operators but that there exists at least one such couple of operators and that it can be constructed that way.

Again, these operators can be illustrated with the example of figure 1: the situation (c) is described by $\mathrm{T}\{$ to $\} \mathrm{B}$ (the truck is tangentially outside of its bumper), $\mathrm{B}\{$ nto $\} \mathrm{H}$ (the bumper is outside the house), in (b) by $\mathrm{B}\{$ to $\} \mathrm{H}$ (the bumper is tangentially outside the house) and in (c) where the bumper does not appear anymore by $\mathrm{T}\{$ to $\} \mathrm{H}$ (the truck is tangentially outside the house). This is compatible 
with the idea that, at a coarser granularity, nto can become to (to $\in \uparrow$ nto) and that at a finer granularity to can become nto (nto $\in \downarrow_{\text {to }}$ ).

The operators obtained for $\mathrm{A}_{2}$ and $\mathrm{A}_{8}$ enjoy the usual properties (including idempotency):

PROPOSITION 14: The upward/downward operators for $A_{2}$ (resp. $A_{8}$ ) of table 11 (resp. table 12) satisfy the properties [1] through [5].

Here, the property [6] is irrelevant because $A_{8}$ is not considered as an interval algebra. A more general property can be established for these kinds of weakening. It states that weakening preserves the properties [1] through [4].

PROPOSITION 15: If the upward/downward operators for $\Gamma$ satisfy the properties [1] through [4], then the upward/downward operators generated as above for one of its weakening $\Gamma^{\prime}$ satisfy them too.

Property [5] (idempotency) is not generally preserved through weakening. For instance, considering a relation algebra with four relations $r_{1}, r_{2}, r_{3}$ and $r_{4}$ such that the (say upward) conversion is $\left\{r_{1}, r_{2}\right\}$ for $r_{1},\left\{r_{3}, r_{4}\right\}$ for $r_{3}$ and identity for the others. If the weakening just groups $r_{2}$ and $r_{3}$ into a new r' relation, then the conversion of $r_{1}$ will then be $\left\{r_{1}, r^{\prime}\right\}$ and applying the conversion once again will yield $\left\{r_{1}, r^{\prime}, r_{4}\right\}$. The same holds true of properties [7], [8] and [9]. They require more constraints on the weakening operation.

\subsection{Discussion: is this sufficient for space ?}

The product constructor has been first presented as a combination of $\mathrm{A}_{13}$ (Güsgen 1989; Mukerjee\& 1990). The results above provide these systems with granularity conversion operators. The products have once been claimed as a nonintuitive representation for space; however, there is some interest in this digital representation of space:

(1) because it is a useful simplification of the representation (Oliver\& 1995),

(2) because it is the representation used by digital sensors such as cameras (Kong\& 1989), and

(3) because it seems to be used in applications like cadastral applications or bounding box calculus (Papadias\& 1995).

Thus the generated operators for granularity conversion could be quite useful in such contexts.

However, this clearly does not account for all the facets of space (the same is true of RCC-8). The research on qualitative space representation is very active. Products can also be used for combining heterogeneous representations (i.e. representations which do not correspond to dimensions of the space). This is exemplified by the system RCC-15 (Cui\& 1993) combining $A_{8}$ between two areas and between their respective convex hull or by (Egenhofer\& 1994) which again combines $A_{8}$ between the generalized regions (insider topological closure of each area) and their respective holes. These products are always followed by a weakening step aimed at eliminating (by grouping them together) the meaningless distinctions (for instance, when the intersection of the closure is empty, the intersection between the holes does not matter; as a matter of fact it is always empty).

The most sophisticated spatial representations are generally obtained by combining different views of the spatial areas (not only extension, borders and direction). The ideal solution would be to consider independently concepts such as 
containment (topology) with orientation (vector spaces) or even distance (metric spaces). For instance, the $A_{15}$ algebra (Zimmermann\& 1993) positioning areas with regard to movement is obtained by the product of $A_{3}$ and $A_{5}$. Other works (Hernández 1994) use pairs of orientations (see figure 4), topological relation and provide the neighborhood structure for this combination. This has been recently extended to a qualitative notion of distance (Clementini\& 1995).
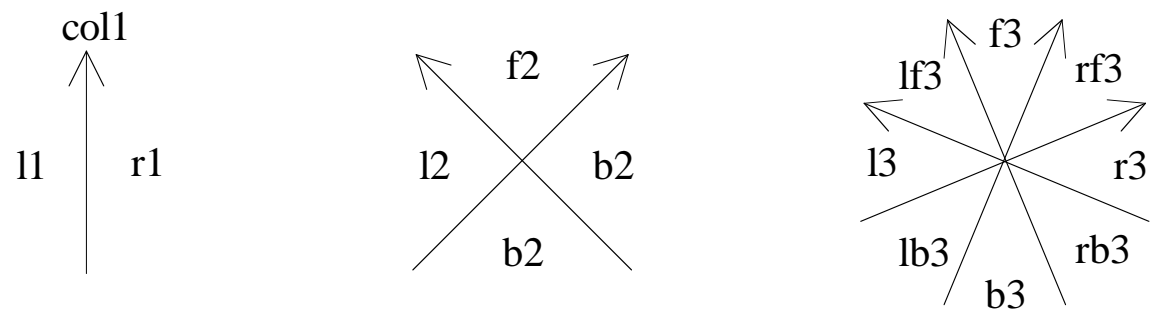

Figure 4 (from (Hernández 1994)): Three sets of directional relationships (from the coarser to the finer).

It seems that such a structure can be given to orientation representation and the existence of granularity operators seems intuitively correct. However, their existence and their possible construction through product and weakening still have to be established.

\section{RELATED WORK}

Jerry Hobbs (1985) introduced the concept of granularity from the nondistinguishability of particular terms with regard to a given set of predicates (these terms can be substituted in the range of any of the predicates without changing their validity). His work has been extended further in (Greer\& 1989) using two partial orders upon which granularity can be based. In the present paper, the granularity has been incorporated a priori in the structure of the algebra, in such a way that it can be mapped to a similar structure, whereas the above-mentioned contributions define a granularity with regard to relevant predicates or partial orders. The approaches thus differ in terms of the language (logic vs. algebra) and the characterization of granularity (a posteriori and a priori).

A tremendous amount of work has been done on granularity in metric spaces. One of the most elaborate models is that of (Montanari\& 1992; Ciapessoni\& 1993). It proposes a quantitative temporal granularity based on a hierarchy of granularities strictly constrained (to be convertible, divisible, etc.) which offers upward and downward conversion operators for instants and intervals (instead of their relationships). (Euzenat 1993) offers a less constrained framework for quantitative relationships and thus achieves weaker properties. Hence, the properties obtained in the present paper for qualitative representation are compatible with the quantitative representation of (Montanari\& 1992; Ciapessoni\& 1993).

Also, a lot of work has been done on calendars and granularity in the database community (e.g. Dyreson\& 1995, Bettini\& 1998a, Cukierman\& 1998) to the point that it has led to the normalization of the vocabulary (Bettini\& 1998b). This work is concerned with an intrinsically metric vision in which the problem is unit conversion, which introduces indeterminacy when converting to a finer granularity. It shares several basic assumptions with the work presented here (such as the definition a priori of conversion through the use of operators) and meets some results (such as those related to granularity and deduction). However, the approach always relies on a quantitative representation where, very often, position variables are replaced by intervals. 
On the qualitative side, various authors have considered granularity (Freksa 1992, Hernández 1994, Grigni\& 1995). They usually consider a new set of relationships which expresses a situation at another (generally coarser) granularity. These sets can form a partition of an initial base set (for instance, by considering $A_{8}$ instead of $A_{13}$ ) or not (consider the three sets of directional relationships of figure 4). On the contrary, the present work uses the same representation formalism at each level of granularity. As a consequence, an arbitrary number of granularity levels can be used instead of a predefined number of such (corresponding to the defined formalism). Only recently, a qualitative model for time granularity has been given (Becher\& 2000). Although, it is expressed in relation algebras, this work is more easily compared with (Montanari 1996) because the various granularities are represented inside the algebra and because objects are converted instead of the relations. The consequence of the former is that, instead of considering several layers made of the point algebra, the authors consider an enhanced point algebra (with 5 relations).

There has been work in granular temporal logics which are not immediately comparable with the present one. (Fiadeiro\& 1994) considers granularity conversions between several representations expressed in the same classical temporal logic (just like here, the same classical algebras have been used). Angelo Montanari (1996) provides a complete account of granularity in a metric temporal logic. Although, a thorough comparison with this work is too far-reaching, it is possible to compare the constraints provided here with axioms of his system. The main difference lies in the qualitative versus quantitative representation, together with the fact that the logic representation converts objects of a temporal domain instead of relationships. However, if one considers that the ordering of objects is a relation, the properties can then be compared:

[1] corresponds to the reflexivity property (but is restricted to the identity conversion, see $\$ 3.2$ ).

[2] does not strictly correspond to something, because there is no specific consideration of neighborhood. However, order preservation is required.

[3] does not strictly correspond to something because there is no specific consideration of converse. However, the equivalent should exist through the displacement operator.

[4] is built-in in the relational representation chosen for the operator.

[5] does not strictly correspond to something because of the quantitative nature of the formalism. It is replaced by several downward/upward transitivity axioms which cover oriented transitivity.

[6] has no equivalent due to the absence of intervals.

At the best of our knowledge, our work is the first one that proposes a general account of granularity in relation algebras. It is even the first one that considers granularity in homogeneous relation algebras for either time or space. A granularitylike conversion operator has been implicitly given for RCC-8 recently (Papadias\& 1995). It consists in converting the RCC-8-relation between two areas into the RCC-8-relation of their minimal bounding rectangles and vice-versa. It appears that the operators built this way are not those of table 14. As a matter of fact, they enjoy properties [1] through [4] but not idempotency [5] (for the same reason as above : the second conversion will yield relations that are not related to the initial relation, but to other forms of an intermediate relation).

\section{CONCLUSION}


In order to understand the relationships between several granularities, a set of requirements has been established for conversion operators. These operators can be used for combining information coming from different sources and overcoming their apparent inconsistency. These constraints have been shown consistent and independent. The only possible operators filling these requirements have been identified for the temporal algebras of points and of relations. Moreover other properties of the operators have been established: preservation of the relationship between points and intervals and non-distributivity of temporal composition over granularity conversion.

The proposed approach is quite unusual. From a very simple formalism $\left(\mathrm{A}_{3}\right)$ the operators have been exported to the more complex one $\left(\mathrm{A}_{13}\right)$ through the particular relationships they enjoy. Then, through other general constructions, namely weakening and product, it has been shown how to find the operators for other formalisms including $\mathrm{A}_{8}$ or $\mathrm{A}_{169}$ (i.e. the orthogonal product of $\mathrm{A}_{13}$ ).

The presented work can be developed in several directions. The first one consists in linking qualitative granularity operators to metric logics (Montanari 1996). The theory presented here is only axiomatic and its semantics still has to be formally provided. This might be obtained by having the same models for granularity in both metric logics and qualitative algebras. The second working path consists in answering two particular questions: what are the conditions which warrant existence and uniqueness of granularity conversion operators for a particular structure? Where is the separation between structures which enjoy distributivity of granularity conversion over composition and those which do not? Finally, we are concerned with the development of a space representation system which is able to process transformations of representations (Buisson\& 1994) and we would like to introduce in it granularity conversion operators and representation conversion operations (such as weakening).

\section{ACKNOWLEDGMENT}

Sections 2 and 3 have partly been presented at the international joint conference on artificial intelligence (IJCAI) at Montréal, 1995 (Euzenat 1995a). Many thanks to the anonymous reviewers who suggested changes in the presentation of the paper.

\section{REFERENCES}

Allen, J. 1983. Maintaining knowledge about temporal intervals. Communication of the ACM, 26(11):832-843.

Badaloni, S., M. Berati. 1994. Dealing with time granularity in a temporal planning system. Lecture notes in computer science, 827:101-116.

Becher, G., F. Clérin-Debart and P. Enjalbert. 2000. A qualitative model for time granularity. Computational intelligence, 16(2):138-168.

Bettini, C., X. Wang and S. Jajodia. 1998a. A general framework for time granularity and its application to temporal reasoning. Annals of mathematics and artificial intelligence, 22(1):29-58.

Bettini, C., C. Dyreson, W. Evans, R. Snodgrass and X. Wang. 1998b. A glossary of time granularity concepts. Lecture notes in computer science, 1399:406-413. 
Buisson, L., and V. Cligniez. 1994. Bases de connaissances spatiales pour l'environnement: le projet ARSEN. Revue internationale de géomatique, 4(34):385-401.

Ciapessoni, E., E. Corsetti, A. Montanari, and P. San Pietro. 1993. Embedding time granularity in a logical specification language for synchronous real-time systems. Science of computer programming, 20(1):141-171.

Clementini, E., P. Di Felice, and D. Hernández. 1995. Qualitative representation of positional information. Artificial intelligence, 95(2):317-356.

Cui, Z., A. Cohn, and D. Randell. 1993. Qualitative and topological relationships. Lecture notes in computer science, 692:296-315.

Cukierman, D., J. Delgrande. 1998. Expressing time intervals and repetition within a formalization of calendars. Computational intelligence, 14(4):563-596.

Dyreson, C., R. Snodgrass. 1995. Temporal granularity. In : R. Snodgrass (ed.). The TSQL2 query language. Kluwer, Amsterdam (NL). 1995.

Egenhofer, M., and R. Franzosa. 1991. Point set topological spatial relations. International journal of geographical information systems, 5(2):161-174.

Egenhofer, M., and K. Al-Taha. 1992. Reasoning about gradual changes of topological relationships. Lecture notes in computer science, 639:192-219.

Egenhofer, M., E. Clementini, and P. Di Felice. 1994. Topological relations between regions with holes. International journal of geographical information systems, 8(2):129-142.

Euzenat, J. 1993. Représentation granulaire du temps. Revue d'intelligence artificielle, 7(3):329-361.

Euzenat, J. 1994. Granularité dans les représentations spatio-temporelles. Technical report 2242, INRIA, Grenoble, FR.

Euzenat, J. 1995a. An algebraic approach to granularity in qualitative space and time representation. Proceedings of the fourteenth international joint conference on artificial intelligence. Montréal, CA, pp. 894-900.

Euzenat, J. 1998. Algèbres d'intervalles sur des domaines temporels arborescents. Proceedings of the eleventh "reconnaissance des formes et intelligence artificielle" conference, Clermont-Ferrand, FR. pp. III-385-394.

Fiadeiro, J. L., and T. Maibaum. 1994. Sometimes "tomorrow" is "sometime": action refinement in a temporal logic of objects. Lecture notes in computer science, 827:48-66.

Freksa, C. 1992. Temporal reasoning based on semi-intervals. Artificial intelligence, 54(1):199-227.

Greer, J., and G. McCalla. 1989. A computational framework for granularity and its application to educational diagnosis. Proceedings of the eleventh international joint conference on artificial intelligence. Detroit, MI US, pp. 477-482.

Grigni, M., D. Papadias, and C. Papadimitriou. 1995. Topological inference. Proceedings of the fourteenth international joint conference on artificial intelligence. Montréal, CA, pp. 901-906. 
Güsgen, H.-W. 1989. Spatial reasoning based on Allen's temporal logic. Research report TR-89-049, International computer science institute, Berkeley, CA US.

Hernández, D. 1994. Qualitative representation of spatial knowledge. (Lecture notes in computer science, 804). Springer, Berlin, DE.

Hirsch, R. 1996. Relation algebras of intervals. Artificial intelligence, 83(2):267-295.

Hobbs, J. 1985. Granularity. Proceedings of the ninth international joint conference on artificial intelligence. Los Angeles, CA US, pp. 432-435.

Kong, T., and A. Rosenfeld. 1989. Digital topology: introduction and survey. CVGIP, 48:357-393.

Ligozat, G. 1990. Weak representations of interval algebras. Proceedings of the eight conference of the american association for artificial intelligence. Boston, MA US, pp. $715-720$.

Montanari, A., E. Maim, E. Ciapessoni, and E. Ratto. 1992. Dealing with time and granularity in the event calculus. Proceedings of the fourth international conference on fifth generation computer systems. Tokyo, JP, pp. 702-712.

Montanari, A. 1996. Metric and layered temporal logic for time granularity. ILLC Dissertation Series 1996-02, University of Amsterdam, Amsterdam, NL.

Mukerjee, A., and G. Joe. 1990. A qualitative model for space. Proceedings of the eight conference of the american association for artificial intelligence. Boston, MA US, pp. 721-727.

Nökel, K. 1988. Convex relations between time intervals. Research report 17. SEKI, Kaiserslautern, DE.

Olivier, P., A. Ormsby, and K. Nakata. 1995. Using occupancy arrays for kinematic reasoning. Proceedings of the IJCAI workshop on "spatial and temporal reasoning". Montréal, CA, pp. 65-74.

Papadias, D., Y. Theodoridis, T. Sellis, and M. Egenhofer. 1995. Topological relations in the world of minimum bounding rectangles: a study with R-trees. Sigmod record, 24(2):92-103.

Randell, D., Z. Cui, and A. Cohn. 1992a. A spatial logic based on regions and connection. Proceedings of the third international conference on principles of knowledge representation and reasoning. Cambridge, MA US, pp. 165-176.

Randell, D., A. Cohn, and Z. Cui. 1992b. Computing transitivity tables: a challenge for automated theorem provers. Lecture notes in computer science, 607:786790.

Tarski A. 1941. On the calculus of relations. Journal of symbolic logic 6(3):73-89.

Vilain, M., and H. Kautz. 1986. Constraint propagation algorithms for temporal reasoning, Proceedings of the fifth conference of the american association for artificial intelligence. Philadelphia, PA US, pp. 377-479.

Zimmermann, K., and C. Freksa. 1993. Qualitative spatial reasoning using orientation, distance and path knowledge, Proceedings of the IJCAI workshop on "spatial and temporal reasoning". Chambéry FR. 


\section{APPENDiX A: NOTATIONS}

These notations are used for the notions of instant and interval relation algebras (Allen 1983), unordered point and area algebras (Egenhofer\& 1992; Randell\& 1992), conceptual neighborhood (Freksa 1992a) and extensions such as the conversion of relations between intervals into points:

$x, y, z \quad$ Temporal and spatial entities (instants, intervals, etc.).

$\mathrm{A}_{3} \quad$ Set of point relations: $\{<,=,>\}$

$\mathrm{A}_{13} \quad$ Set of interval relations: $\left\{\mathrm{b}, \mathrm{d}, \mathrm{o}, \mathrm{s}, \mathrm{f}, \mathrm{m}, \mathrm{e}, \mathrm{m}^{-1}, \mathrm{f}^{-1}, \mathrm{~s}^{-1}, \mathrm{o}^{-1}, \mathrm{~d}^{-1}, \mathrm{~b}^{-1}\right\}$

$\mathrm{A}_{2} \quad$ Set of non ordered point relations: $\{=, \neq\}$

$\mathrm{A}_{8} \quad$ Set of non ordered spatial relations: $\left\{\right.$ nto,to,po,ti,nti,eq, $\left.\mathrm{ti}^{-1}, \mathrm{nti}^{-1}\right\}$

$\Gamma \quad$ Any set of relations.

$2^{\Gamma} \quad$ The power-set of $\Gamma$.

$r, r^{\prime}, r^{\prime \prime}, r_{i} \quad$ Elements of $\Gamma$.

$\rho, \rho, \rho ", \rho_{i} \quad$ Subsets of $\Gamma$.

$r^{-1} \quad$ Converse relationship; extended to sets of relations $\left(\rho^{-1}\right)$.

$r \times_{\Gamma} r \quad$ Composition of relations; extended to sets of relations $\left(\rho \times_{\Gamma} \rho^{\prime}\right)$.

$N_{\Gamma}^{X}\left(r, r^{\prime}\right) \quad \mathrm{X}$-neighborhood relationship on the set $\Gamma$; also used as $N_{\Gamma}^{X}(r)$.

$\Gamma^{n} \quad$ Cartesian product of a set of relations.

$r \times^{n} r^{\prime} \quad$ Extension of a composition operator over the Cartesian product.

$\Rightarrow \quad$ Transformation from interval to instant couples $(\Rightarrow x)$ and interval relations to quadruples of instant relations $(\Rightarrow \mathrm{r})$; extended to sets $(\Rightarrow \rho)$.

$\Leftarrow \quad$ Transformation from instant couples to interval $(\Leftarrow(x, y))$ and quadruples of instant relations to interval relations $\left(\Leftarrow\left(\mathrm{r}_{1}, \mathrm{r}_{2}, \mathrm{r}_{3}, \mathrm{r}_{4}\right)-\right.$ as far as it is possible); extended to sets.

Composition of functions.

These notations are newly introduced in the present article:

$\mathrm{g}, \mathrm{g}$, g" Granularities.

${ }_{\mathrm{g}} \mathrm{g}^{\prime} r \quad$ Upward granularity conversion operator from $\mathrm{g}$ to $\mathrm{g}$; ; extended to sets $\left(g^{\top} g^{\prime} \rho\right)$.

$\mathrm{g}^{\prime} \downarrow_{\mathrm{g}} r \quad$ Downward granularity conversion operator from $\mathrm{g}$ to g'; extended to sets $\left(g^{\prime} \downarrow_{g} \rho\right)$.

$\mathrm{g} \rightarrow \mathrm{g}^{\prime} r \quad$ Generic granularity conversion operator from $\mathrm{g}$ to $\mathrm{g}$; ; extended to

$\begin{array}{ll}\Downarrow_{\Gamma} & \text { sets }\left(\mathrm{g} \rightarrow \mathrm{g}^{\prime} \rho\right) \text {. } \\ & \text { Weakening operator. }\end{array}$

$\Uparrow \Gamma \quad$ Strengthening operator.

$\underset{i=1}{n} \quad$ Product operator. 


\section{APPENDIX B: PROOFS}

Section 4 aims at providing evidence of propositions 4 to 7 . The proofs of the propositions 4 to $10(\$ 4)$ are provided by exhaustive check in (Euzenat 1994). The proofs of propositions 1, 2, $3(\$ 3), 11,12,13$ and $15(\$ 5)$ are given below. Proposition 14 can be easily proof-checked exhaustively. All the properties can be proved by considering a single relationship ( $r$ ) instead of a set of relationships $(\rho)$. Hence, some of them are only proved for a single relationship. The proofs for inverse compatibility are only given in one way because they remain valid when inverting the $\uparrow \downarrow$ operators.

\section{Existence results for algebras of binary relations}

PROPOSITION 1: The algebra based on two elements a and $\mathrm{a}^{-1}$ such that $N\left(\mathrm{a}, \mathrm{a}^{-1}\right)$ has no granularity conversion operators other than identity and non-informative map.

proof. Once given the granularity conversion for a, the conversion for $\mathrm{a}^{-1}$ can be easily deduced through the conversion-reciprocity distributivity. For any possible choice of the conversion operator, there are only two possible values for the conversion of a (because self-preservation constrains the value to contain a itself): $\{a\}$ and $\left\{a, a^{-1}\right\}$. These two values satisfy idempotency and neighborhood compatibility. Inverse compatibility constrains the opposite conversion to be $\{a\}$ in the case of $\{a\}$, and $\left\{a, a^{-1}\right\}$ in that of $\left\{a, a^{-1}\right\}$. On the one hand, if one conversion of $a$ is $\left\{a, a^{-1}\right\}$ then its opposite must contain a for both $a$ and $a^{-1}$ (thus it must be $\left\{a, a^{-1}\right\}$ for $\mathrm{a}^{-1}$, and so for a). This is the non-informative map. On the other hand, if the conversion of $\mathrm{a}$ is $\{\mathrm{a}\}$, and because we just proved that the opposite conversion cannot be $\left\{a, a^{-1}\right\}$, it can only be $\{a\}$ and this satisfies inverse compatibility too. This is identity.

PROPOSITION 2: Given an extended relation algebra containing at least two relationships $\mathrm{a}$ and $\mathrm{b}$ such that neighborhood is the total relation on the relationships, there exists a couple of upward/downward granularity operators defined by:

if $\mathrm{a}$ and $\mathrm{b}$ are auto-inverse: $\downarrow \mathrm{a}=\{\mathrm{a}, \mathrm{b}\}, \uparrow \mathrm{b}=\{\mathrm{a}, \mathrm{b}\}$ the remainder being identity;

if a only is auto-inverse: $\downarrow a=\left\{a, b, b^{-1}\right\}, \uparrow b=\{a, b\}, \uparrow b^{-1}=\left\{a, b^{-1}\right\}$, the remainder being identity;

if $\mathrm{a}$ and $\mathrm{b}$ are not auto-inverse: $\downarrow \mathrm{a}=\{\mathrm{a}, \mathrm{b}\}, \uparrow \mathrm{b}=\{\mathrm{a}, \mathrm{b}\}, \downarrow^{-1}=\left\{\mathrm{a}^{-1}, \mathrm{~b}^{-1}\right\}, \uparrow^{-1}=\left\{\mathrm{a}^{-1}\right.$, $\left.\mathrm{b}^{-1}\right\}$, the remainder being identity. 
proof. It must be noted first that all the given operators trivially satisfy selfconservation and neighborhood compatibility. The conversion-reciprocity distributivity is easily checked in the first case. It deserves a more careful examination for the two others but it appears that it holds. Idempotency is based on the property that the result of any conversion here is either identity (trivially idempotent) or the union of identity convertible relations and the initial relation itself. So applying once again the operator will give the same result. Inverse compatibility is the less obvious property. For the first and the third case, it is based on the same pattern: the conversion of a relation is the relation itself (which satisfies inverse compatibility because of self-preservation of the opposite conversion) or the union of the relation itself with another relation whose opposite conversion yields the exact same result. As a consequence, the inverse compatibility holds for these relations too. Concerning the second case, the downward conversion of a is a plus two other relations whose upward conversion contains a plus themselves, inverse compatibility is satisfied by both operators. The proposed operators are thus valid.

PROPOSITION 3: The core properties of granularity operators are independent..

proof. This is proved by providing set of extended algebras and functions which satisfy all the properties but one. They are provided below, it is easy to check the satisfaction and non satisfaction of properties.

self-conservation) The algebra is based on $a, a^{-1}, b\left(=b^{-1}\right)$ and $c\left(=c^{-1}\right)$; the neighborhood structure is the complete graph. $\uparrow a=\{a, b, c\}, \uparrow b=\{c\}, \uparrow c=$ $\{\mathrm{c}\}, \uparrow^{-1}=\left\{\mathrm{b}, \mathrm{c}, \mathrm{a}^{-1}\right\}, \downarrow \mathrm{a}=\{\mathrm{a}\}, \downarrow \mathrm{b}=\left\{\mathrm{a}, \mathrm{a}^{-1}\right\}, \downarrow \mathrm{c}=\left\{\mathrm{a}, \mathrm{b}, \mathrm{c}, \mathrm{a}^{-1}\right\}$ and $\downarrow^{-1}=\left\{\mathrm{a}^{-1}\right\}$.

neighborhood compatibility) The algebra is based on a, $\mathrm{a}^{-1}, \mathrm{~b}$ and $\mathrm{b}^{-1}$; the neighborhood is restricted to $N\left(\mathrm{a}, \mathrm{a}^{-1}\right), N\left(\mathrm{a}^{-1}, \mathrm{~b}\right), N\left(\mathrm{~b}, \mathrm{~b}^{-1}\right), N\left(\mathrm{~b}^{-1}, \mathrm{a}\right) . \uparrow \mathrm{a}=\{\mathrm{a}, \mathrm{b}\}, \uparrow \mathrm{b}^{-}$ $1=\left\{b^{-1}\right\}, \uparrow b=\{b\}, \uparrow^{-1}=\left\{b^{-1}, a^{-1}\right\}, \downarrow a=\{a\}, \downarrow b^{-1}=\left\{b^{-1}, a^{-1}\right\}, \downarrow b=\{a, b\}$ and $\downarrow \mathrm{a}^{-1}=\left\{\mathrm{a}^{-1}\right\}$.

conversion-reciprocity distributivity) The algebras contains a and $\mathrm{a}^{-1}$ and the neighborhood structure is the complete graph. $\uparrow a=\left\{a, a^{-1}\right\}$ and $\uparrow a^{-1}=\left\{a^{-1}\right\}$, $\downarrow_{\mathrm{a}}=\{\mathrm{a}\}$ and $\downarrow_{\mathrm{a}^{-1}}=\left\{\mathrm{a}, \mathrm{a}^{-1}\right\}$.

inverse compatibility) The algebra is based on a, $\mathrm{a}^{-1}$ and $\mathrm{b}\left(=\mathrm{b}^{-1}\right)$; the neighborhood structure is the complete graph. $\uparrow a=\{a, b\}, \uparrow b=\{b\}, \uparrow^{-1}=\left\{a^{-}\right.$ $\left.{ }^{1}, \mathrm{~b}\right\}, \downarrow \mathrm{a}=\{\mathrm{a}\}, \downarrow \mathrm{b}=\{\mathrm{b}\}$ and $\downarrow_{\mathrm{a}^{-1}}=\left\{\mathrm{a}^{-1}\right\}$.

idempotency) The algebra is based on $a, a^{-1}$ and $b\left(=b^{-1}\right)$; the neighborhood relates only a to $b$ and $a^{-1}$ to $b . \uparrow a=\{a, b\}, \uparrow b=\left\{a, b, a^{-1}\right\}, a^{-1}=\left\{b, a^{-1}\right\}, \downarrow a=\{a, b\}$, $\downarrow_{\mathrm{b}}=\left\{\mathrm{a}, \mathrm{b}, \mathrm{a}^{-1}\right\}$ and $\downarrow_{\mathrm{a}^{-1}}=\left\{\mathrm{b}, \mathrm{a}^{-1}\right\}$.

\section{Product of the interval algebra}


PROPOSITION 11: If the upward/downward operators for $\Gamma$ satisfy the properties [1] through [5], then the upward/downward operators for $\Gamma^{n}$ satisfy them too.

proof.

(self-conservation)

$$
\begin{array}{cr}
\rho & \begin{array}{r}
\text { (definition of } \left.\Gamma^{n}\right) \\
\subseteq \bigcup_{r \in \rho}\left\{\left(r_{1}, \ldots r_{n}\right)\right\}
\end{array} \\
\bigcup_{r \in \rho}\left\{\left(r_{1}^{\prime}, \ldots r_{n}^{\prime}\right)\right\} & \left(\rightarrow \text { definition on } \Gamma^{n}\right) \\
=\bigcup_{r \in \rho} \rightarrow r & \left(\text { extension of } \rightarrow \text { on } \Gamma^{n}\right) \\
=\rightarrow \rho & \diamond
\end{array}
$$

(neighborhood compatibility $\left.{ }^{1}\right)$

$$
\begin{aligned}
& \forall i \in[1 n], \forall r_{i}, \forall s_{i}, t_{i} \in \rightarrow r_{i}, \exists r_{i}^{0}, \ldots r_{i}^{p} \in \rightarrow r_{i} ; r_{i}^{0}=s_{i}, r_{i}^{p}=t_{i} \text { and } \forall j \in[1 p], N\left(r_{i}^{j-1}, r_{i}^{j}\right) \\
& \text { (neighborhood compatibility on } \Gamma \text { ) }
\end{aligned}
$$

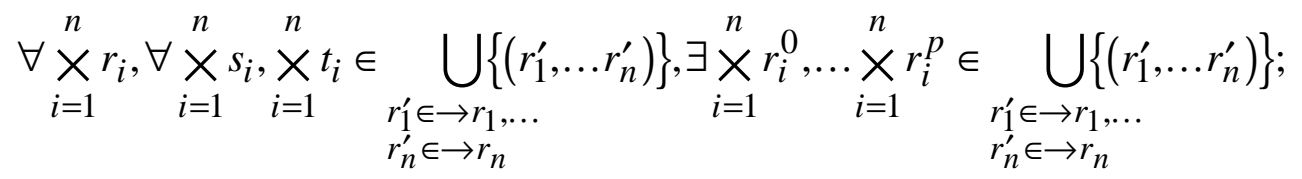

$$
\begin{aligned}
& \underset{i=1}{\times} r_{i}^{0}=\underset{i=1}{\times} s_{i}, \underset{i=1}{\times} r_{i}^{p}=\underset{i=1}{\times} t_{i} \text { and } \forall j \in[1 p], N\left(\underset{i=1}{\underset{\times}{\times}} r_{i}^{j}, \underset{i=1}{\stackrel{n}{\times}} r_{i}^{j-1}\right) \\
& \text { (definition of } \Gamma^{n} \text { ) } \\
& \forall \underset{i=1}{\times} r_{i}, \forall \underset{i=1}{\times} s_{i}, \underset{i=1}{\times} t_{i} \in \rightarrow \underset{i=1}{\times} r_{i}, \exists \underset{i=1}{\times} r_{i}^{0}, \ldots \underset{i=1}{\times} r_{i}^{p} \in \rightarrow \underset{i=1}{\times} r_{i} \\
& \text { (definition of } N \text { and } \rightarrow \text { on } \Gamma^{n} \text { ) } \\
& \Leftrightarrow \quad \forall r, \forall s, t \in \rightarrow r, \exists r_{0}, \ldots r_{p} \in \rightarrow r ; r_{0}=s, r_{p}=t \text { and } \forall j \in[1 p], N\left(r_{j-1}, r_{j}\right) \\
& \text { (definition of } \Gamma^{n} \text { ) } \\
& \diamond \\
& \left(\rightarrow l^{-1} \text { distributivity }\right) \quad \rightarrow r^{-1} \quad\left({ }^{-1} \text { definition on } \Gamma^{n}\right)
\end{aligned}
$$

\footnotetext{
${ }^{1}$ This is also true of a more restricted definition of neighborhood through product (in which two product relations are in neighborhood if and only if they differ by only one projected relation which are themselves neighbors).
} 


$$
\begin{aligned}
& =\rightarrow \underset{i=1}{\stackrel{n}{\times}} r_{i}^{-1} \quad\left(\rightarrow \text { definition on } \Gamma^{n}\right) \\
& =\underset{i=1}{\times} \rightarrow r_{i}^{-1} \quad\left(\rightarrow l^{-1} \text { distributivity in } \Gamma\right)
\end{aligned}
$$

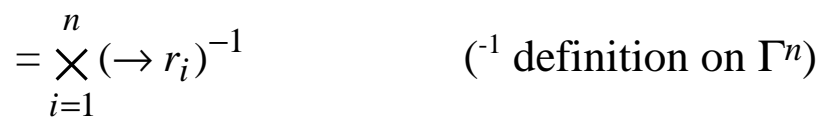

$$
\begin{aligned}
& =\left[\begin{array}{l}
n \\
\underset{i=1}{x} \rightarrow r_{i}
\end{array}\right]^{-1} \quad\left(\rightarrow \text { definition on } \Gamma^{n}\right) \\
& =\left[\rightarrow \underset{i=1}{\times} r_{i}\right]^{-1} \quad\left(\text { definition of } \Gamma^{n}\right) \\
& =(\rightarrow r)^{-1} \quad \diamond
\end{aligned}
$$

(inverse compatibility) $\quad \forall i \in[1 n], r_{i} \in \bigcap_{r_{i}^{\prime} \in \downarrow r_{i}} \uparrow r_{i}^{\prime}$ (inverse compatibility on $\Gamma$ )

$$
\Leftrightarrow \quad \forall i \in[1 n], \forall r_{i}^{\prime} \in \downarrow r_{i}, r_{i} \in \uparrow r_{i}^{\prime} \quad \text { (development) }
$$

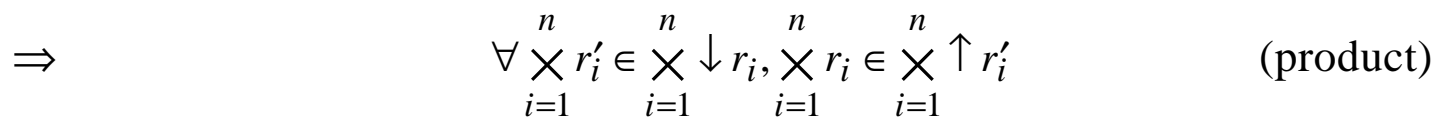$$
\Leftrightarrow \quad \forall \underset{i=1}{\times} r_{i}^{\prime} \in \downarrow \underset{i=1}{\times} r_{i},{ }_{i=1}^{n} r_{i} \in \uparrow \underset{i=1}{\times} r_{i}^{\prime} \quad\left(\rightarrow \text { definition on } \Gamma^{n}\right)
$$$$
\Leftrightarrow \quad r \in \bigcap_{r^{\prime} \in \downarrow_{r}}^{\uparrow r^{\prime}} \quad \diamond
$$$$
\text { (idempotency) } \quad \rightarrow \rightarrow r \quad \text { (definition of } \Gamma^{n} \text { ) }
$$$$
=\rightarrow \rightarrow \underset{i=1}{\stackrel{n}{x}} r_{i} \quad\left(\rightarrow \text { definition on } \Gamma^{n}\right)
$$$$
=\rightarrow \underset{i=1}{\stackrel{n}{x}} \rightarrow r_{i} \quad\left(\rightarrow \text { definition on } \Gamma^{n}\right)
$$

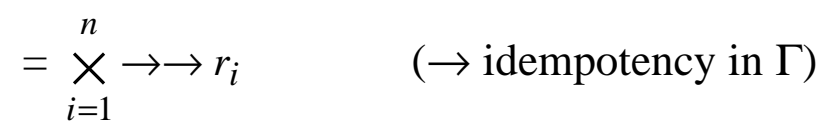$$
=\underset{i=1}{x} \rightarrow r_{i} \quad\left(\rightarrow \text { definition on } \Gamma^{n}\right)
$$$$
\left.=\rightarrow \underset{i=1}{\stackrel{n}{\times}} r_{i} \quad \text { (definition of } \Gamma^{n}\right)
$$$$
\rightarrow r \quad \diamond
$$ 
PROPOSITION 12: Let $\mathrm{H}$ be an algebra of relations and $\Gamma$ one of its interval algebras, if the upward/downward operators for $\Gamma$ satisfy the property [6] with regard to the operators of $\mathrm{H}$, then the upward/downward operators for $\Gamma^{n}$ satisfy the property [6] with regard to the operators of $\mathrm{H}^{n}$.

proof.

$$
\begin{aligned}
& \text { (representation independence 1) } \Rightarrow \rightarrow \Leftarrow \underset{j=1}{4} r^{j} \\
& =\Rightarrow \rightarrow \Leftarrow \underset{j=1}{4} \underset{i=1}{\times} r_{i}^{j} \quad\left(\Leftarrow \text { definition on } \Gamma^{n}\right) \\
& =\Rightarrow \rightarrow \underset{i=1}{n} \Leftarrow{\underset{j=1}{X}}_{j}^{4} r_{i}^{j} \quad\left(\rightarrow \text { definition on } \Gamma^{n}\right) \\
& =\Rightarrow \underset{i=1}{\mathrm{X}} \rightarrow \Leftarrow \underset{j=1}{\times} r_{i}^{j} \quad\left(\Rightarrow \text { definition on } \Gamma^{n}\right) \\
& =\underset{k=1}{\times} \underset{i=1}{\times} \pi_{k} \Rightarrow \rightarrow \Leftarrow \underset{j=1}{4} r_{i}^{j} \\
& \text { (representation independence on } \Gamma \text { ) } \\
& =\underset{k=1}{\times} \underset{i=1}{\times} \pi_{k} \rightarrow \underset{j=1}{\times} r_{i}^{j} \quad\left(\rightarrow \text { definition on } \Gamma^{n}\right) \\
& =\underset{k=1}{\times} \underset{i=1}{\times} \pi_{k} \underset{j=1}{\times} \rightarrow r_{i}^{j} \quad\left(\pi_{k} \text { definition }\right) \\
& =\underset{k=1}{\mathrm{X}} \underset{i=1}{\mathrm{n}} \rightarrow r_{i}^{k} \quad\left(\rightarrow \text { definition on } \Gamma^{n} \text {; twice }\right) \\
& =\rightarrow \underset{k=1}{\times} \underset{i=1}{\times} r_{i}^{k} \quad \text { (definition on } \Gamma^{n} \text { ) } \\
& =\rightarrow \underset{k=1}{\times} r^{k}
\end{aligned}
$$$$
\text { (representation independence 2) } \Leftarrow \rightarrow \Rightarrow r
$$$$
=\Leftarrow \rightarrow \underset{i=1}{\stackrel{n}{\times}} r_{i}
$$$$
\left(\Rightarrow \text { definition on } \Gamma^{n}\right)
$$$$
4 n
$$$$
=\Leftarrow \rightarrow \times \times \pi_{j} \Rightarrow r_{i} \quad\left(\rightarrow \text { definition on } \Gamma^{n} \text {; twice }\right)
$$$$
j=1 i=1
$$$$
=\Leftarrow \underset{j=1}{\operatorname{4}} \underset{i=1}{\mathrm{n}} \rightarrow \pi_{j} \Rightarrow r_{i} \quad\left(\Leftarrow \text { definition on } \Gamma^{n}\right)
$$ 


$$
\begin{aligned}
& =\underset{i=1}{\stackrel{n}{x}} \Leftarrow \underset{j=1}{\stackrel{4}{X}} \rightarrow \pi_{j} \Rightarrow r_{i} \quad\left(\rightarrow \text { definition on } \Gamma^{n}\right) \\
& \left(\pi_{k} \text { definition }\right)
\end{aligned}
$$

$=\stackrel{n}{\times} \Leftarrow \rightarrow \Rightarrow r_{i}$

(representation independence on $\Gamma$ )

$$
=\underset{i=1}{\stackrel{n}{\times}} \rightarrow r_{i}
$$

$\left(\rightarrow\right.$ definition on $\left.\Gamma^{n}\right)$

PROPOSITION 13: The upward/downward operators for $\Gamma^{n}$ satisfy the same properties as $\Gamma$ with regard to properties [7], [8] and [9].

proof.

$(\rightarrow$ distributivity over $\times)$

$$
=\rightarrow\left(\rho \times \rho^{\prime}\right) \quad\left(\text { extension of } \times \text { on } \Gamma^{n}\right)
$$$$
=\rightarrow \bigcup_{r \in \rho, r^{\prime} \in \rho^{\prime}} \bigcup_{r_{1}^{\prime \prime} \in r_{1} \times r_{1}^{\prime}, \ldots r_{n}^{\prime \prime} \in r_{n} \times r_{n}^{\prime}}\left\{\left(r_{n}^{\prime \prime}, \ldots, r_{n}^{\prime \prime}\right)\right\}\left(\text { extension of } \rightarrow \text { on } \Gamma^{n}\right)
$$$$
\left.\left.=\bigcup_{r \in \rho, r^{\prime} \in \rho^{\prime}} \rightarrow \underset{r_{1}^{\prime \prime} \in r_{1} \times r_{1}^{\prime}, \ldots r_{n}^{\prime \prime} \in r_{n} \times r_{n}^{\prime}}{ } \bigcup_{n}^{\prime \prime}\right)\right\}\left(\text { extension of } \rightarrow \text { on } \Gamma^{n}\right)
$$$$
=\bigcup_{r \in \rho, r^{\prime} \in \rho^{\prime}} \bigcup_{r_{1}^{\prime \prime} \in \rightarrow\left(r_{1} \times r_{1}^{\prime}\right), \ldots r_{n}^{\prime \prime} \in \rightarrow\left(r_{n} \times r_{n}^{\prime}\right)}\left\{\left(r_{1}^{\prime \prime}, \ldots r_{n}^{\prime \prime}\right)\right\}(\rightarrow \text { distributivity over } \times)
$$$$
R \bigcup_{r \in \rho, r^{\prime} \in \rho^{\prime}} \bigcup_{r_{1}^{\prime \prime} \in \rightarrow r_{1} \times \rightarrow r_{1}^{\prime}, \ldots r_{n}^{\prime \prime} \in \rightarrow r_{n} \times \rightarrow r_{n}^{\prime}}\left\{\left(r_{1}^{\prime \prime}, \ldots r_{n}^{\prime \prime}\right)\right\}\left(\text { extension of } \times \text { on } \Gamma^{n}\right)
$$$$
=\bigcup_{r \in \rho, r^{\prime} \in \rho^{\prime}}\left[\bigcup_{r_{1}^{\prime \prime} \in \rightarrow r_{1}, \ldots r_{n}^{\prime \prime} \in \rightarrow r_{n}}\left\{\left(r_{1}^{\prime \prime}, \ldots r_{n}^{\prime \prime}\right)\right\}\right] \times\left[\bigcup_{r_{1}^{\prime \prime} \in \rightarrow r_{1}^{\prime}, \ldots r_{n}^{\prime \prime} \in \rightarrow r_{n}^{\prime}}\left\{\left(r_{1}^{\prime \prime}, \ldots r_{n}^{\prime \prime}\right)\right\}\right]
$$

$\left(\right.$ extension of $\times$ on $\left.\Gamma^{n}\right)$

$$
\begin{gathered}
{\left[\bigcup_{r \in \rho} \bigcup_{r_{1}^{\prime \prime} \in \rightarrow r_{1}, \ldots r_{n}^{\prime \prime} \in \rightarrow r_{n}}\left\{\left(r_{n}^{\prime \prime}, \ldots r_{n}^{\prime \prime}\right)\right\}\right] \times\left[\bigcup_{r^{\prime} \in \rho^{\prime}} \bigcup_{r_{1}^{\prime \prime} \in \rightarrow r_{1}^{\prime}, \ldots r_{n}^{\prime \prime} \in \rightarrow r_{n}^{\prime}}\left\{\left(r_{n}^{\prime \prime}, \ldots r_{n}^{\prime \prime}\right)\right\}\right]} \\
\left(\rightarrow \text { definition in } \Gamma^{n}\right) \\
=\left[\bigcup_{r \in \rho} \rightarrow r\right] \times\left[\bigcup_{r^{\prime} \in \rho^{\prime}} \rightarrow r^{\prime}\right] \quad\left(\text { extension of } \rightarrow \text { on } \Gamma^{n}\right) \\
=\rightarrow \rho \times \rightarrow \rho^{\prime},
\end{gathered}
$$

The super and sub distributivity is established through the $R$ relationship between the two terms. This relation can be $=, \supseteq$ or $\subseteq$ depending on the considered algebra $\Gamma$.

\section{Weakening of relation algebras}


LEMMA (monotony of weakening): If $\rho \subseteq \rho^{\prime}$ then $\Downarrow \rho \subseteq \Downarrow \rho^{\prime}$

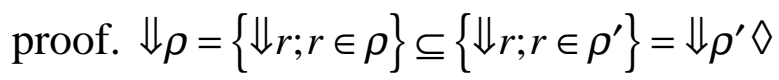

COROLLARY (sub-commutativity of $\Downarrow$ over $\cap$ ): $\Downarrow \bigcap_{\rho \in T} \rho \subseteq \bigcap_{\rho \in T} \Downarrow_{\rho}$

LEMMA (commutativity of $\left.{ }^{-1} / \Uparrow \downarrow\right): \Downarrow_{\rho^{-1}}=(\Downarrow \rho)^{-1}$ and $\Uparrow r^{-1}=(\Uparrow r)^{-1}$.

proof.

(commutativity of ${ }^{-1} / \Uparrow$ )

$$
\begin{aligned}
& \Uparrow \mathrm{r}^{-1} \quad\left({ }^{-1} \text { definition in } \Gamma^{\prime}\right) \\
& =\Uparrow \Downarrow(\Uparrow r)^{-1} \quad\left(\text { closedness of } \Gamma / \Uparrow \text { through }^{-1}\right) \\
& =(\Uparrow r)^{-1} \quad \diamond \\
& \text { (commutativity of } \left.{ }^{-1} / \Downarrow\right) \quad \Downarrow_{\mathrm{r}^{-1}} \text { [w1] } \\
& \left.=\Downarrow \Uparrow\left(\Downarrow_{\mathrm{r}}\right)^{-1} \quad \text { (commutativity of }{ }^{-1} / \Uparrow\right) \\
& =\Downarrow\left(\Uparrow \Downarrow_{\mathrm{r}}\right)^{-1} \quad\left({ }^{-1} \text { definition in } \Gamma^{\prime}\right) \\
& =\left(\Downarrow_{r}\right)^{-1}
\end{aligned}
$$

PROPOSITION 15: If the upward/downward operators for $\Gamma$ satisfy the properties [1] through [4], then the upward/downward operators for one of its weakening $\Gamma$, satisfy them too.

proof.

(self-conservation)

$$
\begin{array}{cr} 
& \rho \\
=\Downarrow \Uparrow \rho & {[\text { w1] }} \\
\subseteq \Downarrow \rightarrow \Uparrow \rho & (\text { self-conservation in } \Gamma) \\
=\rightarrow \rho & \left(\rightarrow \text { definition in } \Gamma^{\prime}\right)
\end{array}
$$

(neighborhood compatibility)

$\forall \mathrm{r}, \mathrm{r}^{\prime} \in \rightarrow \mathrm{r}$

$\left(\rightarrow\right.$ definition in $\left.\Gamma^{\prime}\right)$

$$
\exists \mathrm{s}, \mathrm{s}^{\prime} \in \rightarrow \Uparrow \mathrm{r} ; \mathrm{s} \in \Uparrow \mathrm{r}, \mathrm{s}^{\prime} \in \Uparrow \mathrm{r}^{\prime} \quad \text { (self-conservation in } \Gamma \text { ) }
$$

$\Rightarrow \quad \exists \mathrm{t}, \mathrm{t}^{\prime} \in \Uparrow \mathrm{r} ; \mathrm{s} \in \rightarrow \mathrm{t}, \mathrm{s}^{\prime} \in \rightarrow \mathrm{t}^{\prime}$

(neighborhood compatibility in $\Gamma+[\mathrm{w} 1]$ ) 
738

$$
\begin{aligned}
& \Rightarrow \quad \wedge\left[\exists t_{0}^{\prime}, \ldots t_{m}^{\prime} \in \rightarrow t^{\prime} ; t_{0}^{\prime}=t^{\prime} \wedge t_{m}^{\prime}=s^{\prime} \wedge \forall i \in[1 m], N\left(t_{i-1}^{\prime}, t_{i}^{\prime}\right)\right] \\
& \wedge\left[\Downarrow_{\left.t=\Downarrow_{t^{\prime}}=r\right]}\right. \\
& \left(\begin{array}{c}
\forall i \in[0 n], s_{i}=t_{i} \\
\forall i \in[n+1 n+m+1], s_{i}=t_{i-n-1}^{\prime}
\end{array}\right) \begin{array}{l}
\exists s_{0}, \ldots s_{n+m+1} \in \rightarrow \Uparrow r ; s_{0}=s \wedge s_{n+m+1}=s^{\prime} \\
\Rightarrow \quad \wedge \forall i \in[1 n+m+1],\left[N\left(s_{i-1}, s_{i}\right) \vee \Downarrow_{s_{i-1}}=\Downarrow_{s_{i}}\right]
\end{array}
\end{aligned}
$$

(application of $\Downarrow$ and simplification)

$\Rightarrow \quad \exists r_{0}, \ldots r_{p} \in \rightarrow r ; r_{0}=r \wedge r_{p}=r^{\prime} \wedge \forall i \in[1 p], N^{\prime}\left(r_{i-1}, r_{i}\right)$

$(-1 / \rightarrow$ distributivity)

$$
\begin{aligned}
& (\rightarrow \rho)^{-1} \\
& \left({ }^{-1} \text { and } \rightarrow \text { definition in } \Gamma^{\prime}\right) \\
& =\Downarrow(\Uparrow \Downarrow \rightarrow \Uparrow \rho)^{-1} \\
& (-1 / \Uparrow \Downarrow \text { commutativity) } \\
& =\Downarrow \Uparrow \Downarrow(\rightarrow \Uparrow \rho)^{-1} \\
& (-1 / \rightarrow \text { commutativity in } \Gamma) \\
& =\Downarrow \Uparrow \Downarrow \rightarrow(\Uparrow \rho)^{-1} \\
& \left({ }^{-1} / \Uparrow\right. \text { commutativity) } \\
& =\Downarrow \Uparrow \downarrow \rightarrow \Uparrow \rho^{-1} \\
& \text { [w1] } \\
& =\Downarrow \rightarrow \Uparrow \rho^{-1} \\
& =\rightarrow \rho^{-1} \\
& \left(\rightarrow \text { definition in } \Gamma^{\prime}\right) \\
& r \\
& =\Downarrow \Uparrow r \quad \text { (inverse compatibility in } \Gamma \text { and }
\end{aligned}
$$

(inverse compatibility)

monotony of weakening)

$$
\begin{aligned}
& \left.\subseteq \Downarrow \bigcap_{r^{\prime} \in \uparrow \Uparrow r} \downarrow r^{\prime} \text { (sub-commutativity of } \Downarrow \text { over } \cap\right) \\
& \subseteq \underset{\bigcap^{\prime} \in \uparrow \Uparrow r}{\Downarrow \downarrow \downarrow r^{\prime}} \quad \text { [w3] } \\
& \subseteq \underset{r^{\prime} \in \Downarrow \uparrow \Uparrow r}{\Downarrow \downarrow \downarrow \Uparrow r^{\prime}} \\
& \quad=\bigcap_{r^{\prime} \in \uparrow r} \downarrow r^{\prime}
\end{aligned}
$$




\section{LIST OF FIGURE CAPTIONS}

Figure 1. The same scene at three different granularities. It is taken as a spatial metaphor for granularity and is used throughout the paper.

Figure 2. Neighborhood graphs for (a) instant-to-instant relations, (b) interval-to-interval relations (from (Nökel 1988)). The neighborhood graph is made of relations as nodes and conceptual neighborhood as edges (converse relationships are denoted with an "i" added at the end for the sake of readability).

Figure 3. Transposition of Ligozat (1990) framework on the A-neighborhood graph. Each node is labeled with the number of endpoints that its relationship constrains (resp. does not constrain) when one of the intervals is given. The upward (resp. downward) conversion operator is obtained by gathering, for each relation, the reachable nodes by following a path with strictly ascending labels.

Figure 4 (from (Hernández 1994)): Three sets of directional relationships (from the coarser to the finer). 


\section{LIST OF TABLE CAPTIONS}

Table 1. The 3 relationships between instants $x$ and $y$.

Table 2. Composition table between instant relationships.

Table 3 (from (Allen 1983)). The 13 relationships between two intervals $x$ and $y$.

Table 4. The 13 relationships between intervals expressed through relationships between interval endpoints.

Table 5. Each column represents a possible conversion for $=$ and each row represents a possible conversions for $<$. Because the conversion for $>$ is constrained by [3] to be the converse of that of <, it is not considered here. Thus, each cell represents a conversion operator (defined on the base relations). It contains the numbers corresponding to constraints violated by the corresponding operator (before the backslash, those violated by <; after it, those violated by the conversion of $=$ ).

Table 6. The six possible conversion operators for $=$ and $<$.

Table 7. From the 6 possible operators satisfying individual properties [1], [2] and [3], 36 couples of upward/downward operators can be considered. Among them only the 18 cells marked with an $\bullet$ are compatible with the first equation of property [4]. But, because the compatibility must also be true in the reverse order, only the couples compatibles in both orders remain.

Table 8. Upward and downward granularity conversions between instants.

Table 9. Transformations of upward and downward operators between instants into interval relation quadruples.

Table 10. Upward and downward conversion operators between intervals.

Table 11. Conversion operators for topological spatial relationship between points $\left(\mathrm{A}_{2}\right)$.

Table 12. Conversion operators for topological spatial relationship between areas $\left(\mathrm{A}_{8}\right)$. 